Mon. Not. R. Astron. Soc. 000, ??-?? (2014) Printed 29 January $2021 \quad$ (MN LATEX style file v2.2)

\title{
HCT/HESP study of two carbon stars from the LAMOST survey *
}

\author{
J. Shejeelammal ${ }^{1}$, Aruna Goswami ${ }^{1}$, Jianrong $\mathrm{Shi}^{2}$, \\ ${ }^{1}$ Indian Institute of Astrophysics, Koramangala, Bangalore 560034, India; aruna@iiap.res.in \\ 2 CAS Key Laboratory of Optical Astronomy, National Astronomical Observatories, Beijing 100101, China.
}

Accepted —; Received —; in original form -

\begin{abstract}
Carbon stars, enhanced in carbon and neutron-capture elements, provide wealth of information about the nucleosynthesis history of the Galaxy. In this work, we present the first ever detailed abundance analysis of carbon star LAMOSTJ091608.81+230734.6 and a detailed abundance analysis of neutron-capture elements for the object LAMOSTJ151003.74+305407.3. Updates on the abundances of elements C, O, Mg, Ca, $\mathrm{Cr}, \mathrm{Mn}$ and $\mathrm{Ni}$ for LAMOSTJ151003.74+305407.3 are also presented. Our analysis is based on high resolution spectra obtained using Hanle Echelle Spectrograph (HESP) attached to the Himalayan Chandra Telescope (HCT), IAO, Hanle. The stellar atmospheric parameters $\left(\mathrm{T}_{\text {eff }}\right.$, logg, micro-turbulance $\zeta$, metallicity $\left.[\mathrm{Fe} / \mathrm{H}]\right)$ are found to be $(4820,1.43,1.62,-0.89)$ and $(4500,1.55,1.24,-1.57)$ for these two objects respectively. The abundance estimates of several elements, C, N, O, Na, $\alpha$-elements, Fe-peak elements and neutron-capture elements Rb, Sr, Y, Zr, Ba, La, Ce, Pr, Nd, Sm and $\mathrm{Eu}$ are presented. Our analysis shows the star LAMOSTJ151003.74+305407.3 to be a CEMP-r/s star, and LAMOSTJ091608.81+230734.6 a CH giant. We have examined if the i-process model yields $([\mathrm{X} / \mathrm{Fe}])$ of heavy elements could explain the observed abundances of the CEMP-r/s star based on a parametric model based analysis. The negative values obtained for the neutron density dependent $[\mathrm{Rb} / \mathrm{Zr}]$ ratio confirm former low-mass AGB companions for both the stars. Kinematic analysis shows that LAMOSTJ151003.74+305407.3 belongs to the Galactic halo population and LAMOSTJ091608.81+230734.6 to the disc population.
\end{abstract}

Key words: stars:individual - stars: Carbon - stars: Abundances - stars: nucleosynthesis

\section{INTRODUCTION}

Allowing to measure carbon and neutron-capture elements, the atmospheres of the less-evolved low-mass stars form a unique treasure trove of information for the astrophysicists seeking the chemical evolution history of the Galaxy. Thus, studies on the metal-poor stars such as $\mathrm{CH}$ stars (Keenan 1942) with their more metal-poor counterparts, Carbon Enhanced Metal-Poor (CEMP) stars offer the best means to constrain the neutron-capture nucleosynthesis processes, especially the nucleosythesis occurring in the Asymptotic Giant Branch (AGB) stars. The spectra of these peculiar stars show strong $\mathrm{CH}$ and $\mathrm{C}_{2}$ molecular bands and features due to enhanced neutron-capture elements compared to the normal stars. They are characterized by $\mathrm{C} / \mathrm{O}>1$.

The CEMP stars are more metal-poor $([\mathrm{Fe} / \mathrm{H}]<-1)$

* Based on data collected using HCT/HESP than the classical CH stars (Lucatello et al. 2005, Aoki et al. 2007, Abate et al 2016, Hansen et al. 2016a, c) with $[\mathrm{C} / \mathrm{Fe}]>1$ (Beers \& Christlieb 2005, Abate et al. 2016). They were first identified among the Very Metal-Poor stars discovered in the extensive spectroscopic survey to identify a large sample of most metal-poor stars, HK survey (Beers et al. 1985, 1992, 2007 Beers 1999), and later in a number of successive surveys like Hamburg/ESO Survey (HES; Christlieb et al. 2001a, 2001b, Christlieb 2003), Sloan Digital Sky Survey (SDSS; York et al. 2000), Sloan Extension for Galactic Understanding and Exploration (SEGUE; Yanny et al. 2009) etc. A number of other large sky survey programs in the past were also dedicated to identify the Galactic carbon stars, for instance, the First Byurakan Spectral Sky Survey (Gigoyan et al. 1998), the Automatic Plate Measuring survey (Totten \& Irwin 1998, Ibata et al. 2001), infrared objective-prism surveys (Alksnis et al. 2001), Large Sky Area Multi-Object 
Fiber Spectroscopic Telescope (LAMOST) pilot survey (Cui et al. 2012, Deng et al. 2012, Zhao et al. 2012).

Beers \& Christlieb (2005) put forward the first classification scheme for CEMP stars and classified them into different sub-classes, CEMP-s, CEMP-r, CEMP-r/s and CEMP-no depending on the level of enrichment of neutroncapture elements $\mathrm{Ba}$ and $\mathrm{Eu}$. A slight deviation from the original classification schemes have been adopted by several authors (Aoki et al. 2007, Abate et al. 2016, Frebel 2018, Hansen et al. 2019). High-resolution spectroscopic analyses have shown that, at present, about $80 \%$ of the CEMP stars are CEMP-s stars (Aoki et al. 2007) and about half of the CEMP-s stars are CEMP-r/s stars (Sneden et al. 2008, Käppeler et al. 2011, Bisterzo et al. 2011).

$\mathrm{CH}$ stars and CEMP-s/(r/s) stars belong to the mainsequence or giant phase of stellar evolution. Hence, the observed over abundances of the carbon and neutron-capture elements are attributed to an extrinsic origin. In the case of $\mathrm{CH}$ and CEMP-s stars, enriched in s-process elements, the most accepted scenario involves binary mass-transfer from an AGB companion. There exist a number of proposed scenarios for the simultaneous $\mathrm{r}$ - and s- process enrichment observed in CEMP-r/s stars (Jonsell et al. 2006 and references therein); however, none of them could successfully reproduce the observed frequency and high [hs/ls] ratio of CEMP-r/s stars (Abate et al. 2016). An intermediate neutron-capture process (i-process) that operates with neutron densities in between s- and r-process neutron densities had been invoked to explain the observed abundances of CEMP-r/s stars. Hampel et al. (2016, 2019) could successfully reproduce the observed abundance trend of several CEMP-r/s stars considering this production scenario. The i-process was originally proposed by Cowan \& Rose (1977). Among the proposed scenarios for the nucleosynthetic sites of the i-process are, massive $\left(5-10 \mathrm{M}_{\odot}\right)$ super-AGB stars (Doherty et al. 2015; Jones et al. 2016), evolved low-mass stars (Herwig et al. 2011; Hampel et al. 2019), low-mass, low-metallicity $([\mathrm{Fe} / \mathrm{H}] \leq-3)$ stars (Campbell \& Lattanzio 2008; Campbell et al. 2010; Cruz et al. 2013; Cristallo et al. 2016), and Rapidly Accreting White Dwarfs (Herwig et al. 2014; Denissenkov et al. 2017). Clarkson et al. (2018) and Banerjee et al. (2018) have suggested that massive $(\mathrm{M} \geq 20$ $\left.\mathrm{M}_{\odot}\right)$, metal-poor stars could also play a role in the production of i-process elements. In spite of several efforts, large uncertainties still exist regarding the i-process nucleosynthesis and the possible astrophysical sites of its occurrence.(Frebel 2018; Koch et al. 2019).

It has been found from the long-term radial velocity monitoring studies that vast majority of $\mathrm{CH}$ stars (McClure et al. 1980, McClure 1983, 1984, McClure \& Woodsworth 1990, Jorissen et al. 2016) and CEMP-s/rs stars (Lucatello et al. 2005, Starkenburg et al. 2014, Jorissen et al. 2016, Hansen et al. 2016c) are most likely binaries, thus strongly favoring the binary mass transfer scenario.

It has been identified that the fraction of CEMP stars in the Galactic halo increases with decreasing metallicity; $\sim 20 \%$ for $[\mathrm{Fe} / \mathrm{H}]<-2$ (Norris et al. 1997, Rossi et al. 1999, 2005, Christlieb 2003, Cohen et al. 2005, Marsteller et al. 2005, Frebel et al. 2006, Lucatello et al. 2006, Carollo et al. 2012, Lee et al. 2013), $\sim 40 \%$ for $[\mathrm{Fe} / \mathrm{H}]<-3$ (Aoki et al. 2013, Lee et al. 2013, Yong et al. 2013b), $\sim 75 \%$ for $[\mathrm{Fe} / \mathrm{H}]<-4$ (Lee et al. 2013, Placco et al. 2014, Frebel \&
Norris 2015), and thus making them important tools for the studies of formation and evolution of early Galactic halo.

Ji et al. (2016) have identified 894 new carbon stars from the LAMOST DR2 which contains almost four million medium-resolution $(\mathrm{R} \sim 1800)$ stellar spectra, based on multiple line indices measurement. In this work, we have carried out a detailed spectroscopic analysis of two carbon stars LAMOSTJ091608.81+230734.6 and LAMOSTJ151003.74+305407.3 from Ji et al. (2016).

Observations and data reduction are presented in Section 2. Radial velocity of the stars and the methodology used for the determination of stellar atmospheric parameters are presented in section 3 . The same section also provides a brief discussion on the stellar mass determination. Section 4 provides a discussion on abundance uncertainties. Elemental abundance determination is discussed in Section 5. In Section 6, we present the kinematic analysis of the program stars, followed by the discussion on the binary status of the stars in Section 7. Interpretations of abundance ratios are presented in Section 8. A discussion on individual stars along with the parametric model based analysis is also given in section 8. Conclusion are drawn in Section 9.

\section{OBSERVATIONS AND DATA REDUCTION}

High-quality, high-resolution spectra of the objects LAMOSTJ091608.81+230734.6

and LAMOSTJ151003.74+305407.3 were obtained with the HESP (Hanle Echelle SPectrograph) attached to the $2 \mathrm{~m} \mathrm{Hi}$ malayan Chandra Telescope (HCT) operated by Indian Astronomical Observatory, Hanle. The HESP spectra covers the wavelength range 3530 - $9970 \AA$. The spectra of LAMOSTJ091608.81+230734.6 $\left(\mathrm{V}_{\text {mag }}=10.4\right)$ were obtained on April 4, 2018 at a spectral resolution $(\lambda / \delta \lambda) \sim 60,000$, and the spectra of LAMOSTJ151003.74+305407.3 $\left(\mathrm{V}_{\text {mag }}=\right.$ 11.38) were obtained on May 23, 2018 at a spectral resolution $(\lambda / \delta \lambda) \sim 30,000$. For both the objects we had acquired three frames; each frame was taken with 2700 seconds exposure time. The three frames were added to enhance the $\mathrm{S} / \mathrm{N}$ ratio, and the co-added spectrum was used for further analysis. The data was reduced using the standard procedures in IRAF $^{1}$ software. The basic information of the program stars are given in Table 1. Two sample spectra of the stars in the wavelength region 5160 - $5190 \AA$ are shown in Figure 1.

\section{ESTIMATION OF ATMOSPHERIC PARAMETERS AND RADIAL VELOCITY}

A set of clean lines of several elements is used to calculate the radial velocities of the program stars. While LAMOSTJ151003.74+305407.3 is found to be a high velocity object with an estimated radial velocity

1 IRAF (Image Reduction and Analysis Facility) is distributed by the National Optical Astronomical Observatories, which is operated by the Association for Universities for Research in Astronomy, Inc., under contract to the National Science Foundation 
Table 1. Basic information of the program stars.

\begin{tabular}{lccccccccccc}
\hline Star & RA(2000) & Dec.(2000) & B & V & J & H & K & $\begin{array}{c}\text { Exposure } \\
\text { (seconds) }\end{array}$ & Date of obs. & $\begin{array}{c}\text { S/N } \\
5500 \AA\end{array}$ & $7500 \AA$ \\
\hline LAMOSTJ091608.81+230734.6 & 09168.82 & +230734.86 & 11.44 & 10.40 & 8.654 & 8.141 & 8.022 & $2700(3)$ & $04 / 04 / 2018$ & 37.15 & 42.38 \\
LAMOSTJ151003.74+305407.3 & 15103.30 & +30547.36 & 13.50 & 11.38 & 9.33 & 8.737 & 8.539 & $2700(3)$ & $23 / 05 / 2018$ & 33.67 & 70.17 \\
\hline
\end{tabular}

The number of frames taken are indicated within the parenthesis with exposure.

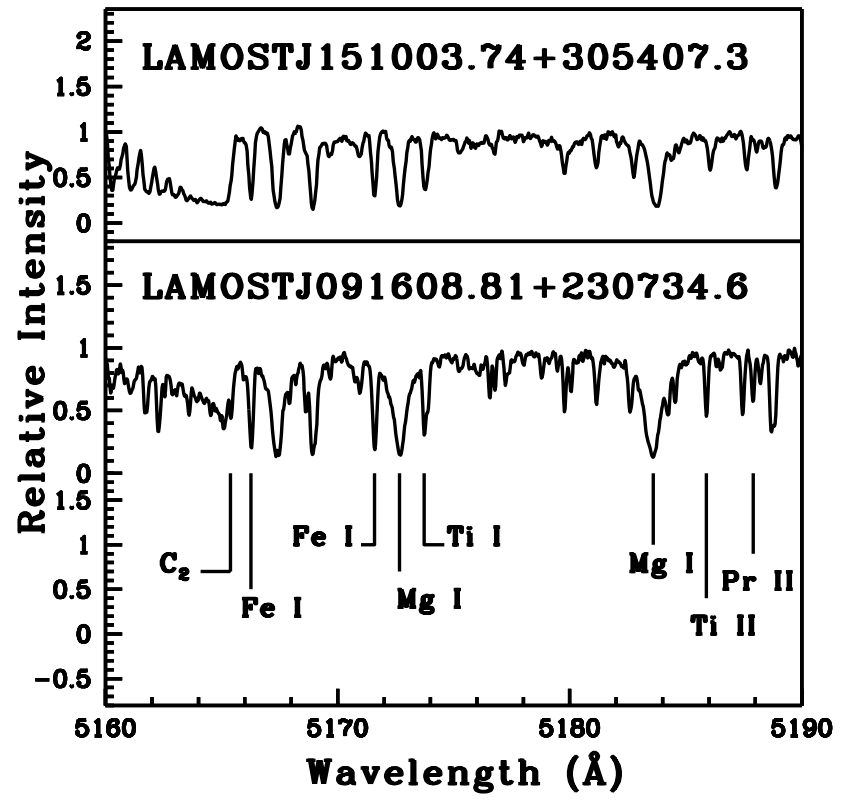

Figure 1. Sample spectra of the program stars in the wavelength region 5160 to $5190 \AA$.

of $-141.58 \pm 3.57 \mathrm{kms}^{-1}$, the radial velocity of LAMOSTJ091608.81+230734.6 is found to be $16.13 \pm 4.30 \mathrm{kms}^{-1}$.

The radial velocities of these two objects are -145.2 and $17.3 \mathrm{kms}^{-1}$ respectively, as noted from the SIMBAD astronomical database (Gaia collaboration et al. 2018). For the star LAMOSTJ151003.74+305407.3, our estimate shows a difference of $\sim 4 \mathrm{kms}^{-1}$ from the SIMBAD value; this may be a clear indication that the star could be a binary.

The equivalent width measurements of a set of Fe I and Fe II lines are used to derive the atmospheric parameters of the stars. The lines are selected such that the range of equivalent width and excitation potential are $20-180$ $\mathrm{m} \AA$ and 0.0 - $6.0 \mathrm{eV}$ respectively. The photometric temperature estimates, estimated using the temperature calibration equations of Alonso et al. (1999, 2001), had been used as an initial guess to derive the stellar atmospheric parameters. The final model atmosphere is obtained through an iterative process from the initial one taken from the $\mathrm{Ku}-$ rucz grid of model atmosphere with no convective overshooting (http://cfaku5.cfa.hardvard.edu/). We made use of the recent version MOOG2013 of the radiative transfer code MOOG (Sneden 1973) assuming Local Thermodynamic Equilibrium (LTE) for the analysis.

The temperature which gives nearly zero slope between the abundance and excitation potential of Fe I lines is taken as the effective temperature. The microturbulent velocity at this fixed effective temperature is then determined such that

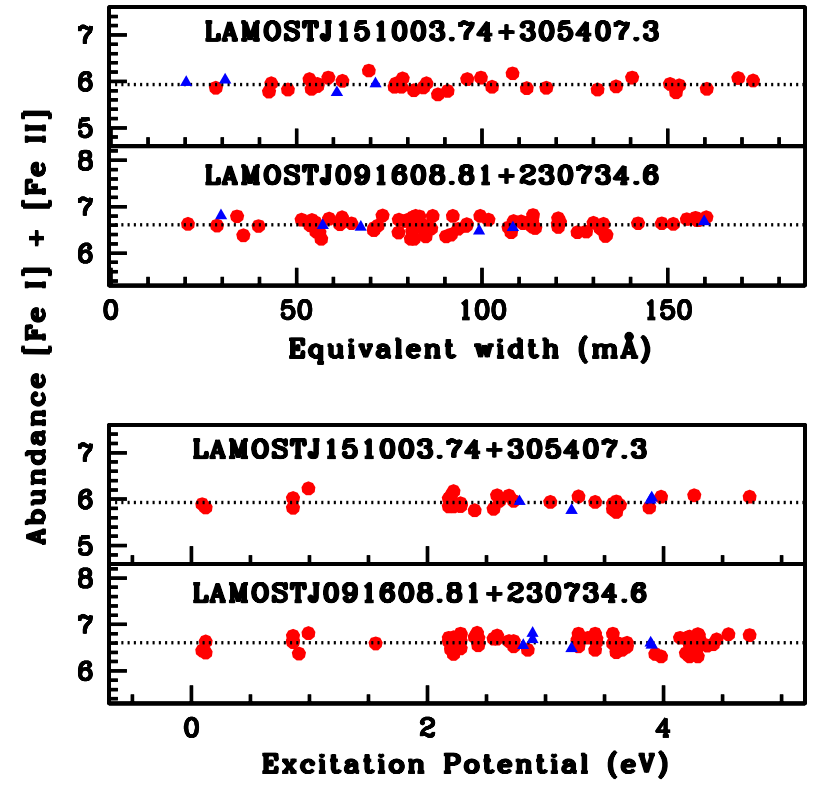

Figure 2. Iron abundances of the program stars derived from individual Fe I and Fe II lines as function of (i) Excitation potential (lower panel), (ii) equivalent width (upper panel). Solid circles correspond to Fe I and solid triangles correspond to Fe II lines.

there is no dependence of the Fe I abundance on equivalent width. With this temperature and microturbulent velocity estimates, the surface gravity is determined by demanding the abundances obtained from Fe I and Fe II lines are to be nearly same. Figure 2 shows the abundances estimated from Fe I and Fe II lines, as functions of excitation potential and equivalent widths. The derived atmospheric parameters and the radial velocity estimates are given in Table 2 .

We have determined the mass of the star LAMOSTJ151003.74+305407.3 from its position in the Hertzsprung-Russell diagram, generated using the evolutionary tracks of Girardi et al. 2000, with the estimates of spectroscopic temperature, $\mathrm{T}_{\mathrm{eff}}$, and the luminosity, $\log \left(L / L_{\odot}\right)$. Then $\log g$ is recalculated using this mass estimate as described in our previous work Shejeelammal et al. (2020). For estimation of $\log \left(L / L_{\odot}\right)$, the required visual magnitudes $\mathrm{V}$ and the parallaxes $\pi$ are taken from Simbad and Gaia DR2 (Gaia collaboration et al. 2018, https://gea.esac.esa.int/archive/) respectively. We have used $\mathrm{z}=0.001$ tracks for this star. The evolutionary tracks for LAMOSTJ151003.74+305407.3 is shown in Figure 3 . The estimated mass, log g determined using parallax method are presented in Table 3 . The mass could not be determined for the other star using this method as the evo- 
Table 2. Derived atmospheric parameters of the program stars.

\begin{tabular}{|c|c|c|c|c|c|c|c|c|}
\hline Star & $\begin{array}{l}\mathrm{T}_{\mathrm{eff}} \\
(\mathrm{K}) \\
\pm 100\end{array}$ & $\begin{array}{l}\log g \\
\operatorname{cgs} \\
\pm 0.2\end{array}$ & $\begin{array}{c}\zeta \\
\left(\mathrm{km} \mathrm{s}^{-1}\right) \\
\pm 0.2\end{array}$ & {$[\mathrm{Fe} \mathrm{I} / \mathrm{H}]$} & {$[\mathrm{Fe} \mathrm{II/H}]$} & $\begin{array}{c}\mathrm{V}_{r} \\
\left(\mathrm{~km} \mathrm{~s}^{-1}\right) \\
\text { our estimates }\end{array}$ & $\begin{array}{c}\mathrm{V}_{r} \\
\left(\mathrm{~km} \mathrm{~s}^{-1}\right) \\
\text { SIMBAD }\end{array}$ & Remarks \\
\hline LAMOSTJ091608.81+230734.6 & 4820 & 1.43 & 1.62 & $-0.89 \pm 0.14$ & $-0.89 \pm 0.12$ & $+16.13 \pm 4.30$ & $+17.33 \pm 0.40$ & 1 \\
\hline LAMOSTJ151003.74+305407.3 & 4500 & 1.55 & 1.24 & $-1.57 \pm 0.12$ & $-1.57 \pm 0.12$ & $-141.58 \pm 3.57$ & $-145.25 \pm 0.003$ & 1 \\
\hline & 4358.31 & 0.956 & 1.667 & -1.346 & - & - & - & 2 \\
\hline
\end{tabular}

Remarks: 1. Our work, 2. Hayes et al. (2018)

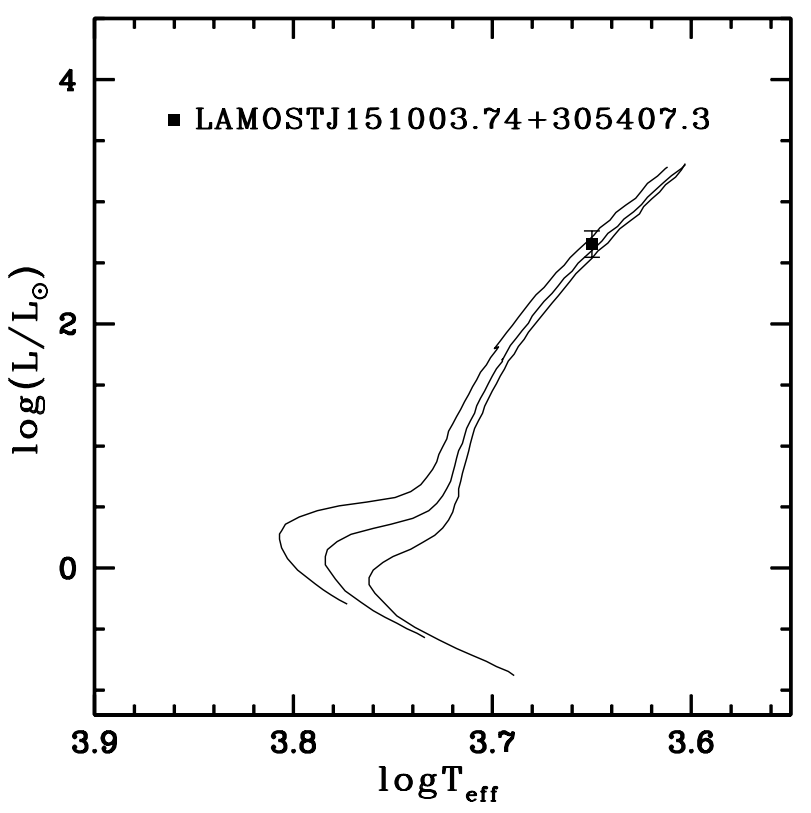

Figure 3. The locations of LAMOSTJ151003.74+305407.3. The evolutionary tracks for $0.6,0.7$ and $0.8 \mathrm{M}_{\odot}$ are shown from bottom to top for $\mathrm{z}=0.001$.

lutionary tracks corresponding to its temperature and luminosity are not available.

\section{ABUNDANCE UNCERTAINTIES}

The main sources of uncertainty in the abundance are the errors in the estimated stellar atmospheric parameters and the errors in the line parameters. Total uncertainty in the elemental abundance, $\log \epsilon$, is given as;

$$
\begin{gathered}
\sigma_{\text {log } \epsilon}^{2}=\sigma_{\text {ran }}^{2}+\left(\frac{\partial \log \epsilon}{\partial T}\right)^{2} \sigma_{T_{e f f}}^{2}+\left(\frac{\partial \log \epsilon}{\partial \log g}\right)^{2} \sigma_{\text {logg }}^{2}+ \\
\left(\frac{\partial \log \epsilon}{\partial \zeta}\right)^{2} \sigma_{\zeta}^{2}+\left(\frac{\partial \log \epsilon}{\partial[\mathrm{Fe} / H]}\right)^{2} \sigma_{[\mathrm{Fe} / H]}^{2}
\end{gathered}
$$

where $\sigma_{\text {ran }}=\frac{\sigma_{s}}{\sqrt{N}}, \sigma_{s}$ being the standard deviation of the abundances derived from $\mathrm{N}$ lines of a particular element considered. The other $\sigma_{s}^{\prime}$ in the equation are the typical uncertainties in the atmospheric parameters; $\Delta \mathrm{T}_{\text {eff }} \sim \pm 100 \mathrm{~K}$, $\Delta \log \mathrm{g} \sim \pm 0.2 \mathrm{dex}, \Delta \zeta \sim \pm 0.2 \mathrm{~km} \mathrm{~s}^{-1}$ and $\Delta[\mathrm{Fe} / \mathrm{H}] \sim \pm 0.1$ dex.

We have calculated the uncertainty in the abundance, $[\mathrm{X} / \mathrm{Fe}]$, for each element following a detailed procedure described in our earlier paper Shejeelammal et al.
(2020). As an example, these values for the star LAMOSTJ091608.81+230734.6 are given in Table 4 .

\section{ABUNDANCE DETERMINATION}

Elemental abundances are derived from the measured equivalent widths and the spectral synthesis calculation of spectral lines of neutral and ionized atoms. Only clean unblended lines have been considered for the abundance determination. Absorption lines due to each element is identified from the close comparison of Doppler-corrected spectrum of the star Arcturus and the program stars spectra. The line information such as $\log g f$ and the lower excitation potential are from the Kurucz database of atomic line lists. In addition to the equivalent width method, spectral synthesis calculation is also performed for the elements showing hyper-fine splitting. Also, the abundances derived from the molecular bands are based on spectral synthesis calculation. The hyper-fine components of Eu are taken from Worely et al. (2013), Ba from Mcwilliam (1998), V, Co and Cu from Prochaska et al. (2000) and Sc and Mn from Prochaska \& Mcwilliam (2000). Solar abundance values are taken from Asplund et al. (2009).

The abundance results are presented in Table 5. The lines used for the estimation of abundances are given in Tables $\mathrm{A} 1$ and $\mathrm{A} 2$.

\subsection{Abundance analysis: $\mathrm{C}, \mathrm{N}, \mathrm{O},{ }^{12} \mathrm{C} /{ }^{13} \mathrm{C}, \mathrm{Na}, \alpha-$ and $F e$-peak elements}

The abundance of oxygen is derived using the spectral synthesis calculation of $[\mathrm{O} \mathrm{I}]$ line at $6300.304 \AA$ in LAMOSTJ091608.81+230734.6. The resonance O I triplet lines at around $7770 \AA$ and the [O I] $6363.776 \AA$ line are blended and could not be used for abundance determination. The estimated oxygen abundance shows near solar value, with $[\mathrm{O} / \mathrm{Fe}] \sim-0.04$. We could not estimate the abundance of oxygen for the star LAMOSTJ151003.74+305407.3 as no clean good lines of oxygen could be detected in the spectrum of this object.

The carbon abundance is derived from the spectral synthesis calculation of the $\mathrm{C}_{2}$ molecular bands at 5165 and $5635 \AA$ (Figure 4). Both the bands gave the same carbon abundance value in both the stars. Carbon is found to be enhanced in LAMOSTJ151003.74+305407.3 with $[\mathrm{C} / \mathrm{Fe}] \sim 1.74$ and mildly enhanced in the other object with $[\mathrm{C} / \mathrm{Fe}] \sim 0.36$.

Once the carbon abundance is estimated, the abundance of nitrogen is derived using the spectral synthesis calculation of ${ }^{12} \mathrm{CN}$ lines at $8000 \AA$ region. The ${ }^{12} \mathrm{CN}$ molecular band at $4215 \AA$ is not usable in both the stars. The CN and 
Table 3. Mass and log g estimates by parallax method

\begin{tabular}{lcccccc}
\hline Star name & $\begin{array}{c}\text { Parallax } \\
(\mathrm{mas})\end{array}$ & $M_{\text {bol }}$ & $\log (\mathrm{L} / \mathrm{L} \odot)$ & $\operatorname{Mass}\left(\mathrm{M}_{\odot}\right)$ & $\begin{array}{c}\log \mathrm{g} \\
(\mathrm{cgs})\end{array}$ & $\begin{array}{c}\log \mathrm{g}(\mathrm{spectroscopic}) \\
(\mathrm{cgs})\end{array}$ \\
\hline LAMOSTJ091608.81+230734.6 & $1.0543 \pm 0.0517$ & $0.105 \pm 0.107$ & $1.854 \pm 0.043$ & - & - & 1.43 \\
LAMOSTJ151003.74+305407.3 & $0.2778 \pm 0.0346$ & $1.891 \pm 0.271$ & $2.653 \pm 0.109$ & $0.70 \pm 0.10$ & $1.20 \pm 0.05$ & 1.55 \\
\hline
\end{tabular}

Table 4. Differential Abundance $(\Delta \log \epsilon)$ of different elemental species due to the variations in stellar atmospheric parameters for LAMOSTJ091608.81+230734.6. The sixth column gives the computed rms uncertainty of the second to fifth columns. The seventh column gives the total uncertainty in the abundance ratio, $[\mathrm{X} / \mathrm{Fe}]$, of each elemental species.

\begin{tabular}{|c|c|c|c|c|c|c|}
\hline Element & $\begin{array}{c}\Delta \mathrm{T}_{\text {eff }} \\
( \pm 100 \mathrm{~K})\end{array}$ & $\begin{array}{c}\Delta \log \mathrm{g} \\
( \pm 0.2 \mathrm{dex})\end{array}$ & $\begin{array}{c}\Delta \zeta \\
\left( \pm 0.2 \mathrm{kms}^{-1}\right)\end{array}$ & $\begin{array}{c}\Delta[\mathrm{Fe} / \mathrm{H}] \\
( \pm 0.1 \mathrm{dex})\end{array}$ & $\left(\Sigma \sigma_{i}^{2}\right)^{1 / 2}$ & $\sigma_{[X / F e]}$ \\
\hline $\mathrm{C}$ & \pm 0.10 & 0.00 & 0.00 & $\mp 0.05$ & 0.11 & 0.19 \\
\hline $\mathrm{N}$ & \pm 0.13 & \pm 0.03 & 0.00 & \pm 0.02 & 0.13 & 0.21 \\
\hline $\mathrm{O}$ & 0.00 & \pm 0.04 & $\mp 0.02$ & 0.00 & 0.04 & 0.16 \\
\hline $\mathrm{Na} \mathrm{I}$ & \pm 0.08 & $\mp 0.01$ & $\mp 0.05$ & $\mp 0.01$ & 0.10 & 0.19 \\
\hline Mg I & \pm 0.09 & $\mp 0.03$ & $\mp 0.08$ & $\mp 0.01$ & 0.05 & 0.17 \\
\hline Si I & \pm 0.04 & \pm 0.01 & $\mp 0.02$ & 0.00 & 0.06 & 0.17 \\
\hline $\mathrm{Ca} \mathrm{I}$ & \pm 0.10 & $\mp 0.01$ & $\mp 0.08$ & $\mp 0.01$ & 0.13 & 0.21 \\
\hline Sc II & $\mp 0.02$ & \pm 0.09 & $\mp 0.06$ & \pm 0.02 & 0.11 & 0.25 \\
\hline $\mathrm{Ti} \mathrm{I}$ & \pm 0.14 & $\mp 0.01$ & $\mp 0.07$ & $\mp 0.01$ & 0.16 & 0.23 \\
\hline Ti II & 0.00 & \pm 0.09 & $\mp 0.08$ & \pm 0.03 & 0.12 & 0.26 \\
\hline V I & \pm 0.16 & $\mp 0.01$ & $\mp 0.07$ & $\mp 0.01$ & 0.18 & 0.24 \\
\hline Cr I & \pm 0.17 & $\mp 0.02$ & $\mp 0.13$ & $\mp 0.03$ & 0.22 & 0.27 \\
\hline Mn I & \pm 0.09 & $\mp 0.02$ & $\mp 0.16$ & $\mp 0.01$ & 0.18 & 0.24 \\
\hline $\mathrm{Fe} I$ & \pm 0.10 & \pm 0.01 & $\mp 0.11$ & \pm 0.05 & 0.16 & - \\
\hline $\mathrm{Fe} \mathrm{II}$ & $\mp 0.06$ & \pm 0.11 & $\mp 0.10$ & $\mp 0.17$ & 0.23 & - \\
\hline Co I & \pm 0.07 & \pm 0.02 & $\mp 0.06$ & \pm 0.01 & 0.09 & 0.18 \\
\hline $\mathrm{Ni} \mathrm{I}$ & \pm 0.09 & 0.00 & $\mp 0.05$ & $\mp 0.01$ & 0.10 & 0.19 \\
\hline $\mathrm{Zn} \mathrm{I}$ & $\mp 0.03$ & \pm 0.05 & $\mp 0.03$ & \pm 0.02 & 0.07 & 0.17 \\
\hline $\mathrm{Rb} \mathrm{I}$ & \pm 0.10 & 0.00 & $\mp 0.03$ & 0.00 & 0.10 & 0.19 \\
\hline Sr I & \pm 0.18 & $\mp 0.02$ & $\mp 0.13$ & $\mp 0.02$ & 0.22 & 0.27 \\
\hline Y I & \pm 0.17 & $\mp 0.02$ & $\mp 0.04$ & $\mp 0.02$ & 0.18 & 0.24 \\
\hline Y II & 0.00 & \pm 0.09 & $\mp 0.11$ & \pm 0.03 & 0.14 & 0.27 \\
\hline Zr I & \pm 0.17 & $\mp 0.02$ & $\mp 0.07$ & $\mp 0.02$ & 0.19 & 0.25 \\
\hline Zr II & \pm 0.01 & \pm 0.08 & $\mp 0.06$ & \pm 0.02 & 0.10 & 0.25 \\
\hline $\mathrm{Ba}$ II & \pm 0.02 & \pm 0.05 & $\mp 0.15$ & \pm 0.02 & 0.16 & 0.28 \\
\hline La II & \pm 0.01 & \pm 0.09 & $\mp 0.06$ & \pm 0.03 & 0.11 & 0.25 \\
\hline Ce II & \pm 0.02 & \pm 0.08 & $\mp 0.13$ & \pm 0.02 & 0.16 & 0.28 \\
\hline Pr II & \pm 0.02 & \pm 0.08 & $\mp 0.07$ & \pm 0.02 & 0.11 & 0.25 \\
\hline Nd II & \pm 0.03 & \pm 0.08 & $\mp 0.16$ & \pm 0.02 & 0.18 & 0.29 \\
\hline Sm II & \pm 0.04 & \pm 0.09 & $\mp 0.08$ & \pm 0.03 & 0.13 & 0.26 \\
\hline $\mathrm{Eu} \mathrm{II}$ & $\mp 0.02$ & \pm 0.09 & $\mp 0.03$ & \pm 0.03 & 0.10 & 0.25 \\
\hline
\end{tabular}


Table 5. Elemental abundances in LAMOSTJ091608.81+230734.6 and LAMOSTJ151003.74+305407.3

\begin{tabular}{|c|c|c|c|c|c|c|c|c|}
\hline & & & \multicolumn{3}{|c|}{ LAMOSTJ091608.81+230734.6 } & \multicolumn{3}{|c|}{ LAMOSTJ151003.74+305407.3 } \\
\hline & $\mathrm{Z}$ & solar $\log \epsilon^{*}$ & $\log \epsilon$ & {$[\mathrm{X} / \mathrm{H}]$} & {$[\mathrm{X} / \mathrm{Fe}]$} & $\log \epsilon$ & {$[\mathrm{X} / \mathrm{H}]$} & {$[\mathrm{X} / \mathrm{Fe}]$} \\
\hline $\mathrm{C}\left(\mathrm{C}_{2}\right.$ band $\left.5165 \AA\right)$ & 6 & 8.43 & $7.90($ syn) & -0.53 & 0.36 & 8.60(syn) & 0.17 & 1.74 \\
\hline $\mathrm{C}\left(\mathrm{C}_{2}\right.$ band $\left.5635 \AA\right)$ & 6 & 8.43 & $7.90(\operatorname{syn})$ & -0.53 & 0.36 & $8.60($ syn) & 0.17 & 1.74 \\
\hline $\mathrm{N}$ & 7 & 7.83 & 7.79 (syn) & -0.04 & 0.85 & $7.53(\mathrm{syn})$ & -0.30 & 1.27 \\
\hline $\mathrm{O}$ & 8 & 8.69 & 7.76(syn) & -0.93 & -0.04 & - & - & - \\
\hline $\mathrm{NaI}$ & 11 & 6.24 & $6.03 \pm 0.15(4)$ & -0.21 & 0.68 & $5.24 \pm 0.05(2)$ & -1.00 & 0.57 \\
\hline $\mathrm{Mg} \mathrm{I}$ & 12 & 7.60 & $7.12 \pm 0.15(2)$ & -0.46 & 0.43 & $6.20 \pm 0.18(3)$ & -1.58 & -0.01 \\
\hline Si I & 14 & 7.51 & $6.59 \pm 0.18(3)$ & -0.92 & -0.03 & - & - & - \\
\hline $\mathrm{Ca} \mathrm{I}$ & 20 & 6.34 & $5.38 \pm 0.15(10)$ & -0.96 & -0.07 & $4.52 \pm 0.13(8)$ & -1.82 & -0.25 \\
\hline Sc II & 21 & 3.15 & $2.19(\operatorname{syn})$ & -0.96 & -0.07 & $1.85(\operatorname{syn})$ & -1.30 & 0.27 \\
\hline Ti I & 22 & 4.95 & $4.07 \pm 0.17(12)$ & -0.88 & 0.01 & $3.30 \pm 0.14(4)$ & -1.65 & -0.08 \\
\hline Ti II & 22 & 4.95 & $3.77 \pm 0.17(4)$ & -1.18 & -0.29 & $3.21 \pm 0.19(5)$ & -1.74 & -0.17 \\
\hline V I & 23 & 3.93 & $3.42(\operatorname{syn})$ & -0.51 & 0.38 & $3.11(\operatorname{syn})$ & -0.82 & 0.75 \\
\hline Cr I & 24 & 5.64 & $5.13 \pm 0.18(6)$ & -0.51 & 0.38 & $3.59 \pm 0.10(5)$ & -2.05 & -0.48 \\
\hline Mn I & 25 & 5.43 & $4.70($ syn $)$ & -0.73 & 0.16 & $3.43(\operatorname{syn})$ & -2.00 & -0.43 \\
\hline Fe I & 26 & 7.50 & $6.61 \pm 0.14(73)$ & -0.89 & - & $5.93 \pm 0.12(35)$ & -1.57 & - \\
\hline Fe II & 26 & 7.50 & $6.61 \pm 0.12(6)$ & -0.89 & - & $5.93 \pm 0.12(4)$ & -1.57 & - \\
\hline Co I & 27 & 4.99 & 3.89(syn) & -1.10 & -0.21 & - & - & - \\
\hline $\mathrm{Ni} \mathrm{I}$ & 28 & 6.22 & $5.63 \pm 0.11(11)$ & -0.59 & 0.30 & $4.61 \pm 0.17(6)$ & -1.61 & -0.04 \\
\hline Zn I & 30 & 4.56 & $4.11(1)$ & -0.49 & 0.40 & $2.56 \pm 0.14(2)$ & -2.00 & -0.43 \\
\hline $\mathrm{Rb} \mathrm{I}$ & 37 & 2.52 & $2.10($ syn $)$ & -0.42 & 0.47 & $1.00(\operatorname{syn})$ & -1.52 & 0.05 \\
\hline Sr I & 38 & 2.87 & $2.95(\mathrm{syn})$ & 0.08 & 0.97 & - & - & - \\
\hline Y I & 39 & 2.21 & 2.56 (syn) & 0.35 & 1.24 & 2.20 (syn) & -0.01 & 1.56 \\
\hline Y II & 39 & 2.21 & $2.32 \pm 0.10(6)$ & 0.11 & 1.00 & $3.05 \pm 0.11(2)$ & 0.84 & 2.40 \\
\hline Zr I & 40 & 2.58 & $3.08(\operatorname{syn})$ & 0.50 & 1.39 & $2.05(\mathrm{syn})$ & -0.53 & 1.04 \\
\hline Zr II & 40 & 2.58 & $2.73(\mathrm{syn})$ & 0.15 & 1.04 & 2.09 (syn) & -0.49 & 1.08 \\
\hline $\mathrm{Ba}$ II & 56 & 2.18 & 2.48 (syn) & 0.30 & 1.19 & 2.00 (syn) & -0.18 & 1.39 \\
\hline La II & 57 & 1.10 & $2.00($ syn) & 0.90 & 1.79 & 1.10 (syn) & 0.00 & 1.57 \\
\hline Ce II & 58 & 1.58 & $2.34 \pm 0.11(7)$ & 0.76 & 1.65 & $1.32 \pm 0.13(7)$ & -0.26 & 1.31 \\
\hline Pr II & 59 & 0.72 & $1.48 \pm 0.18(6)$ & 0.76 & 1.65 & $1.02 \pm 0.11(5)$ & 0.30 & 1.87 \\
\hline $\mathrm{Nd}$ II & 60 & 1.42 & $2.17 \pm 0.17(9)$ & 0.75 & 1.64 & $1.35 \pm 0.14(12)$ & -0.07 & 1.50 \\
\hline Sm II & 62 & 0.96 & $1.41 \pm 0.11(10)$ & 0.45 & 1.34 & $1.03 \pm 0.08(6)$ & 0.07 & 1.64 \\
\hline Eu II & 63 & 0.52 & $0.33(\mathrm{syn})$ & -0.19 & 0.70 & $0.09(\mathrm{syn})$ & -0.43 & 1.14 \\
\hline
\end{tabular}

* Asplund (2009), The numbers within the parenthesis are the number of lines used for abundance estimation.

$\mathrm{C}_{2}$ molecular lines are taken from Ram et al. (2014), Sneden et al. (2014) and Brooke et al. (2013). In both the stars nitrogen is enhanced with $[\mathrm{N} / \mathrm{Fe}] \geq 0.85$. The final $\mathrm{C}, \mathrm{N}$ and $\mathrm{O}$ abundances are determined by an iterative process. Using the first estimate of oxygen abundance derived from the spectral synthesis calculation of $6300.304 \AA[\mathrm{O} \mathrm{I}]$ line, the abundance of carbon is estimated from the $\mathrm{C}_{2}$ molecular bands at 5165 and $5635 \AA$. The abundance of nitrogen is then determined using these derived abundance estimates of $\mathrm{O}$ and $\mathrm{C}$. Once the nitrogen abundance is obtained, the oxygen and carbon abundances are re-determined using this nitrogen abundance. This iteration process is continued until a convergence is reached.

The carbon isotopic ratio, ${ }^{12} \mathrm{C} /{ }^{13} \mathrm{C}$, is estimated from the spectral synthesis calculation of ${ }^{12} \mathrm{CN}$ lines at $8003.292,8003.553,8003.910 \AA$, and ${ }^{13} \mathrm{CN}$ features at $8004.554,8004.728,8004.781 \AA$. The spectrum synthesis fits for the program stars in this region is shown in Figure 5. The values of this ratio are 8.67 and 13.33 in LAMOSTJ091608.81+230734.6 and LAMOSTJ151003.74+305407.3 respectively. These are typical values normally found in the case of giants (Smith et al. 1993). For CEMP-s and CEMP-r/s stars, the values of ${ }^{12} \mathrm{C} /{ }^{13} \mathrm{C}$ ratio are found in the range 2.5 - 40 (Bisterzo et al. 2011). We could estimate the $\mathrm{C} / \mathrm{O}$ ratio in the star LAMOSTJ091608.81+230734.6 which is found to be greater than 1 as typically seen in $\mathrm{CH}$ stars.

The abundances of the elements $\mathrm{Na}, \mathrm{Mg}, \mathrm{Si}, \mathrm{Ca}, \mathrm{Ti}, \mathrm{Cr}$, $\mathrm{Ni}$ and $\mathrm{Zn}$ are derived from the measured equivalent width of spectral lines listed in Table A2. Scandium abundance is derived from the spectral synthesis calculation of Sc II line at $6245.637 \AA$, vanadium abundance from the $\mathrm{V}$ I lines at 6251.827 and $4864.731 \AA$, manganese abundance from Mn I line at $6013.513 \AA$ and the abundance of cobalt from Co I line at $5342.695 \AA$.

A comparison of our estimated light element abundances for the star LAMOSTJ151003.74+305407.3 with the literature values is given in Table 6 . Within the error limits, our estimates of $\mathrm{Mg}$ and $\mathrm{Ni}$ match with the estimates of Hayes et al. (2018). However, our estimates are lower by $\sim 0.6$ dex for $[\mathrm{Ca} / \mathrm{Fe}]$ and $[\mathrm{Cr} / \mathrm{Fe}]$ and $\sim 0.3$ dex for $[\mathrm{Mn} / \mathrm{Fe}]$. We have obtained $[\mathrm{C} / \mathrm{Fe}] \sim 1.74$, which is largely different from $\sim 0.81$ of Hayes et al. (2018).

\subsection{Heavy element abundance analysis}

\subsubsection{The light s-process elements: $\mathrm{Rb}, \mathrm{Sr}, \mathrm{Y}, \mathrm{Zr}$}

The spectral synthesis calculation of resonance line of $\mathrm{Rb} \mathrm{I}$ at $7800.259 \AA$ is used to derive the $\mathrm{Rb}$ abundance in both the stars. The Rb I 7947.597 $\AA$ line was not usable for the abundance estimation. The Rb hyperfine components are taken from Lambert \& Luck (1976). It is mildly enhanced in LAMOSTJ091608.81+230734.6 with $[\mathrm{Rb} / \mathrm{Fe}] \sim 0.47$, while it is near-solar in LAMOSTJ151003.74+305407.3.

We could derive the strontium abundance only in LAMOSTJ091608.81+230734.6 as no usable lines could be measured on the spectrum of the other star. The Sr I 4607.327 $\AA$ line in LAMOSTJ091608.81+230734.6 returned a value $[\mathrm{Sr} / \mathrm{Fe}] \sim 0.97$ from the spectral synthesis calculation.

The abundance of yttrium is derived using the spectral synthesis calculation of Y I line at $6435.004 \AA$ and equivalent 
Table 6. Comparison of the light element abundances of LAMOSTJ151003.74+305407.3 with the literature values.

\begin{tabular}{lccccccc}
\hline Star name & {$[\mathrm{C} / \mathrm{Fe}]$} & {$[\mathrm{Mg} / \mathrm{Fe}]$} & {$[\mathrm{Ca} / \mathrm{Fe}]$} & {$[\mathrm{Cr} / \mathrm{Fe}]$} & {$[\mathrm{Mn} / \mathrm{Fe}]$} & {$[\mathrm{Ni} / \mathrm{Fe}]$} & $\mathrm{Ref}$ \\
\hline LAMOSTJ151003.74+305407.3 & 1.74 & -0.01 & -0.25 & -0.48 & -0.43 & -0.04 & 1 \\
& 0.81 & 0.21 & 0.36 & 0.11 & -0.15 & 0.07 & 2 \\
\hline
\end{tabular}

References: 1. Our work, 2. Hayes et al. 2018
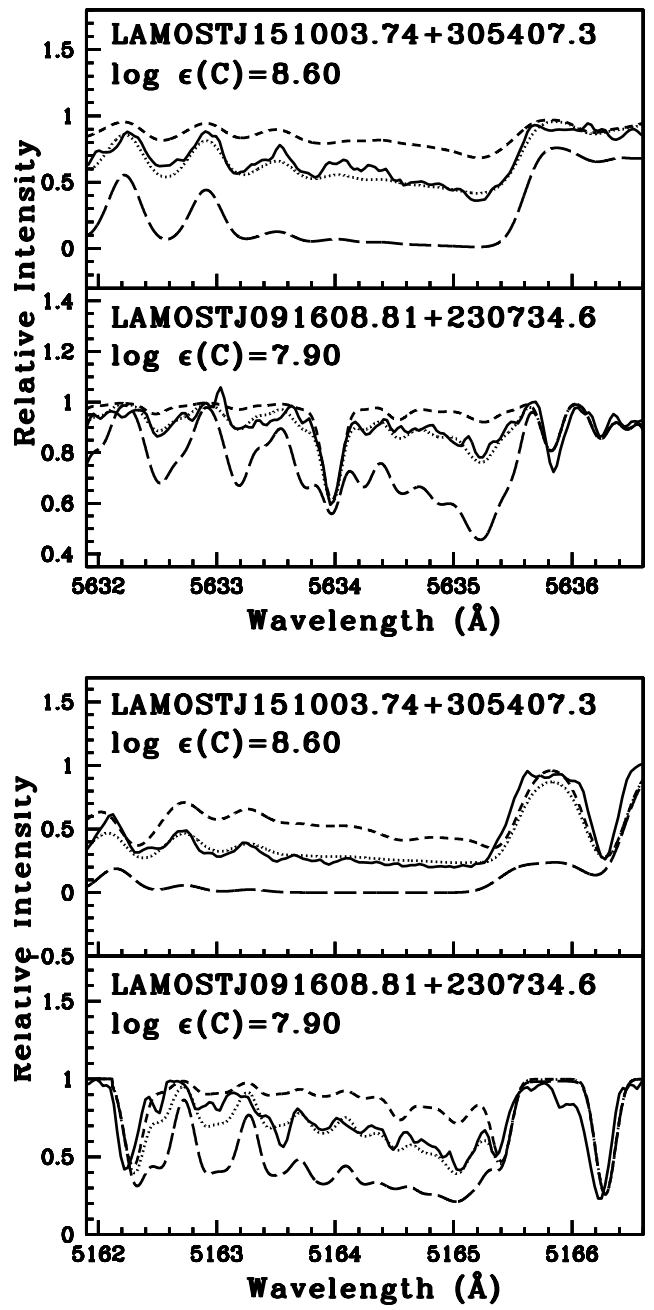

Figure 4. Synthesis of $\mathrm{C}_{2}$ band around $5165 \AA$ (lower panel) and $5635 \AA$ (upper panel). Dotted and solid lines represent synthesized and observed spectra respectively. Short dashed and long dashed lines represent the synthetic spectra for $\Delta[\mathrm{C} / \mathrm{Fe}]=-0.3$ and +0.3 respectively.

width measurement of a few Y II lines in both the stars. Both the species give a value $[\mathrm{Y} / \mathrm{Fe}] \geq 1$. The spectrum synthesis fits for Y I of the program stars are shown in Figure 6.

The spectral synthesis calculation of $\mathrm{Zr}$ I line at $6134.585 \AA$ and Zr II line at $5112.297 \AA$ are used to derive the zirconium abundance in the program stars. In both the cases, $\mathrm{Zr}$ is found to be enhanced with $[\mathrm{Zr} / \mathrm{Fe}]>1$. The spectrum synthesis fits for Zr I of the program stars are shown in Figure 7.

In the case of LAMOSTJ091608.81+230734.6, neutral

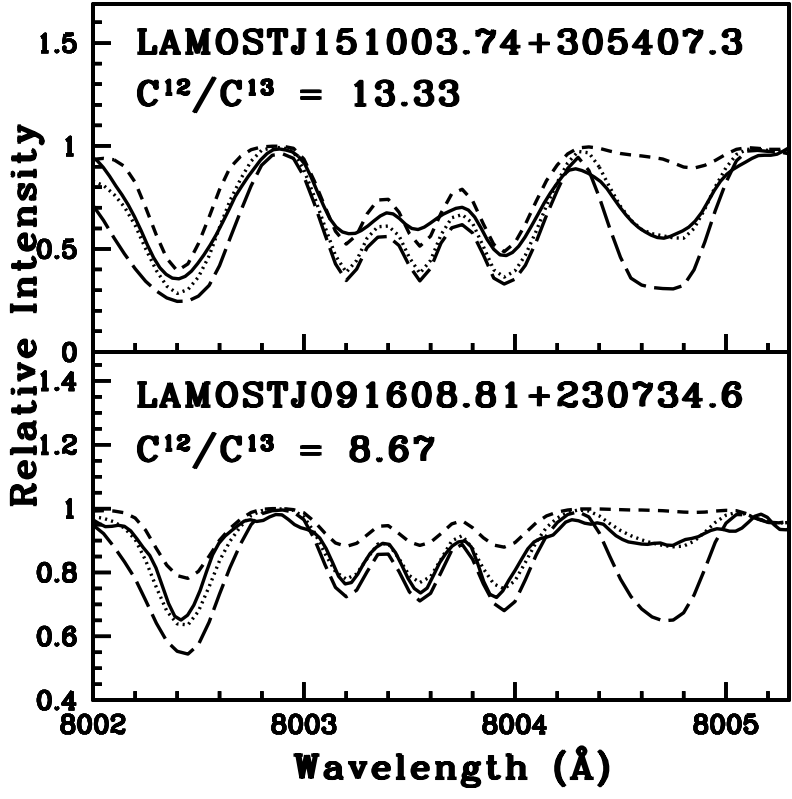

Figure 5. Spectral synthesis of $\mathrm{CN}$ band around $8005 \AA$. Dotted and solid lines represent synthesized and observed spectra respectively. Short dashed and long dashed lines are the synthetic spectra for ${ }^{12} \mathrm{C} /{ }^{13} \mathrm{C} \simeq 83$ and 2.7 respectively.

lines of $\mathrm{Y}$ and $\mathrm{Zr}$ give a higher abundance than the singly ionized lines.

\subsubsection{The heavy s-process elements: $\mathrm{Ba}, \mathrm{La}, \mathrm{Ce}, \mathrm{Pr}$, Nd}

The spectral synthesis calculation of Ba II line at 5853.668 $\AA$ in LAMOSTJ091608.81+230734.6 and Ba II $6141.713 \AA$ line in LAMOSTJ151003.74+305407.3 are used to derive the barium abundances. La abundance is derived from the spectral synthesis calculation of La II line at 5259.380 $\AA$ in both the stars. We could not detect any other useful lines due to lanthanum in the program stars for the abundance determination. The abundances of $\mathrm{Ce}, \mathrm{Pr}$ and $\mathrm{Nd}$ are derived using the measured equivalent widths of several spectral lines due to singly ionized species of the respective elements. All these elements are found to be enhanced in both the stars with $[\mathrm{X} / \mathrm{Fe}]>1$.

Finally, we have estimated the [ls/Fe], [hs/Fe] and [hs/ls] ratios in the program stars. Here, ls and hs are light (Sr, Y and Zr) and heavy (Ba, La, Ce and Nd) s-process elements respectively. Also, we have estimated the mean abundance ratio of s-process elements ( $\mathrm{Sr}, \mathrm{Y}, \mathrm{Zr}, \mathrm{Ba}, \mathrm{La}, \mathrm{Ce}, \mathrm{Nd}$ ), [s/Fe], to find the s-process content. 


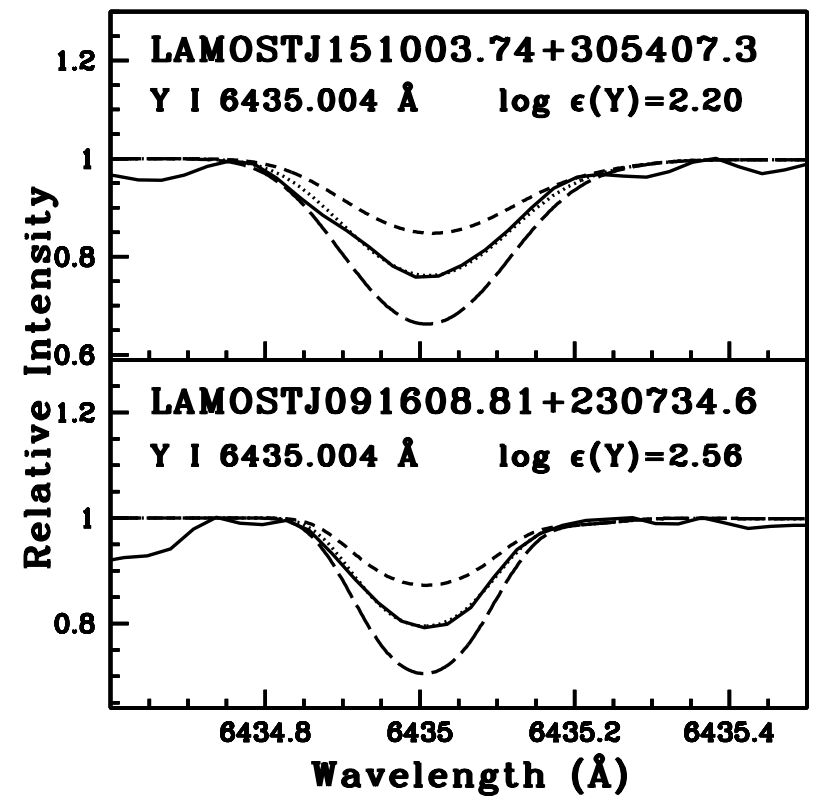

Figure 6. Synthesis of Y I line at $6435.004 \AA$. Dotted and solid lines represent synthesized and observed spectra respectively. Short dashed and long dashed lines represent the synthetic spectra for $\Delta[\mathrm{Y} / \mathrm{Fe}]=-0.3$ and +0.3 respectively.

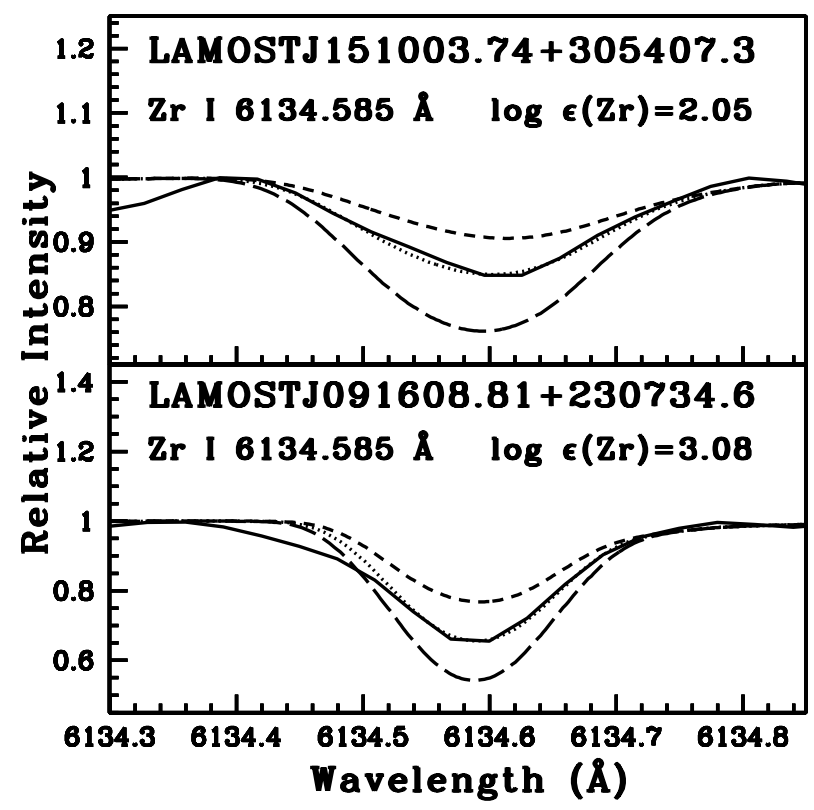

Figure 7. Synthesis of Zr I line at $6134.585 \AA$ A. Dotted and solid lines represent synthesized and observed spectra respectively. Short dashed and long dashed lines represent the synthetic spectra for $\Delta[\mathrm{Zr} / \mathrm{Fe}]=-0.3$ and +0.3 respectively.

\subsubsection{The r-process elements: Sm, Eu}

The Sm abundance is derived from the equivalent width measurement of Sm II lines listed in Table A2. Both the stars show enhancement of $\mathrm{Sm}$ with $[\mathrm{Sm} / \mathrm{Fe}] \sim 1.34$ and 1.64 in LAMOSTJ091608.81+230734.6 and LAMOSTJ151003.74+305407.3 respectively.

The abundance of europium is estimated from the spectral synthesis calculation of $\mathrm{Eu}$ II $6645.064 \AA$. In LAMOSTJ091608.81+230734.6 $\mathrm{Eu}$ is enhanced with $[\mathrm{Eu} / \mathrm{Fe}] \sim 0.70$ while the other star shows a value 1.14 .

\section{KINEMATIC ANALYSIS}

The spatial velocity of a star in the solar neighborhood is measured with respect to the Local Standard of Rest (LSR). The components of the spatial velocity are $U_{L S R}$, $V_{L S R}$ and $W_{L S R}$; measured along the axes pointing towards the Galactic center, the direction of Galactic rotation and the North Galactic Pole respectively (Johnson \& Soderblom 1987). Space velocity of the programme stars are calculated following the procedures in Bensby et al. (2003). Components of the spatial velocity of the star with respect to LSR:

$$
(U, V, W)_{L S R}=(U, V, W)+(U, V, W)_{\odot} \mathrm{km} / \mathrm{s} .
$$

where, $(U, V, W)_{\odot}$ is the solar motion with respect to LSR and its value is $(11.1,12.2,7.3) \mathrm{km} / \mathrm{s}$ (Schönrich et al., 2010) and

$$
\left[\begin{array}{c}
U \\
V \\
W
\end{array}\right]=B \cdot\left[\begin{array}{c}
V_{r} \\
k \cdot \mu_{\alpha} / \pi \\
k \cdot \mu_{\delta} / \pi
\end{array}\right]
$$

where, $B=T . A$, $\mathrm{T}$ is the transformation matrix connecting the Galactic coordinate system and equatorial coordinate system and $\mathrm{A}$ is a coordinate matrix defined below

$$
\begin{aligned}
& T=\left[\begin{array}{ccc}
-0.06699 & -0.87276 & -0.48354 \\
+0.49273 & -0.45035 & +0.74458 \\
-0.86760 & -0.18837 & 0.46020
\end{array}\right] \\
& A=\left[\begin{array}{ccc}
\text { Cos } \alpha . \operatorname{Cos} \delta & -\operatorname{Sin} \alpha & - \text { Cos } \alpha . \operatorname{Sin} \delta \\
\operatorname{Sin} \alpha \cdot \operatorname{Cos} \delta & \operatorname{Cos} \alpha & -\operatorname{Sin} \alpha . \operatorname{Sin} \delta \\
\operatorname{Sin} \delta & 0 & \operatorname{Cos} \delta
\end{array}\right]
\end{aligned}
$$

where $\alpha$ is the RA, $\delta$ the DEC, $V_{r}$ the radial velocity in $\mathrm{km} / \mathrm{s}, k=4.74057 \mathrm{~km} / \mathrm{s}$ equivalent of $1 \mathrm{AU}$ in one year, $\mu_{\alpha}$ and $\mu_{\delta}$ respectively the proper motions in RA and DEC in $\operatorname{arcsec} /$ year and $\pi$ the parallax in arcsec. The proper motion and parallax values are taken from GAIA DR2 (Gaia collaboration et al. 2018, https://gea.esac.esa.int/archive/) and SIMBAD astronomical database. The spectroscopic velocity estimates have been used in this calculation.

The total spatial velocity of the star:

$V_{s p a}^{2}=U_{L S R}^{2}+V_{L S R}^{2}+W_{L S R}^{2}$

Errors in the respective velocity components are calculated as follows:

$$
\begin{aligned}
& {\left[\begin{array}{c}
\sigma_{U}^{2} \\
\sigma_{V}^{2} \\
\sigma_{W}^{2}
\end{array}\right]=C \cdot\left[\begin{array}{c}
(k / \pi)^{2}\left[\sigma_{\mu_{\alpha}}^{2}+\left(\mu_{\alpha} \sigma_{\pi} / \pi\right)^{2}\right] \\
(k / \pi)^{2}\left[\sigma_{\mu_{\delta}}^{2}+\left(\mu_{\delta} \sigma_{\pi} / \pi\right)^{2}\right]
\end{array}\right]+} \\
& \frac{2 \mu_{\alpha} \mu_{\delta} k^{2} \sigma_{\pi}^{2}}{\pi^{4}} \cdot\left[\begin{array}{c}
b_{12} \cdot b_{13} \\
b_{22} \cdot b_{23} \\
b_{32} \cdot b_{33}
\end{array}\right]
\end{aligned}
$$

where $C_{i j}=b_{i j}^{2}$ and $\sigma_{s}^{\prime}$ the errors in respective quantities. 
The probability that a star belongs to the Galactic thin/thick disc or halo population is calculated following the procedure described in Mishenina et al. (2004), Bensby et al. (2003, 2004) and Reddy et al. (2006), with assumption that the space velocities follow a Gaussian distribution.

$$
\begin{aligned}
& P_{\text {thin }}=\frac{f_{1} \cdot p_{1}}{P}, P_{\text {thick }}=\frac{f_{2} \cdot p_{2}}{P}, P_{\text {halo }}=\frac{f_{3} \cdot p_{3}}{P} \\
& P=\Sigma f_{i} \cdot p_{i} \\
& p_{i}=K_{i} \cdot \exp \left[\frac{-U_{L S R}^{2}}{2 \cdot \sigma_{U_{i}}^{2}}-\frac{\left(V_{L S R}-V_{a d}\right)^{2}}{2 \cdot \sigma_{V_{i}}^{2}}-\frac{W_{L S R}^{2}}{2 \cdot \sigma_{W_{i}}^{2}}\right] \\
& K_{i}=\frac{1}{(2 \cdot \pi)^{(3 / 2)} \cdot \sigma_{U_{i}} \cdot \sigma_{V_{i}} \cdot \sigma_{W_{i}}} ; i=1,2,3
\end{aligned}
$$

where $\sigma_{s}^{\prime}$ are the dispersion in velocities, $V_{a d}$ the mean galactic rotation velocity for each stellar population relative to LSR and $f$ the fractional population. These values are taken from Reddy et al. (2006).The estimates of the total spatial velocity and components of spatial velocity along with the probability estimates are given in Table 7. Our analysis shows that the star LAMOSTJ091608.81+230734.6 belongs to the thin disc population and LAMOSTJ151003.74+305407.3 belongs to the Galactic halo population.

\section{BINARY STATUS OF THE PROGRAM STARS}

For a precise identification of the source of carbon and heavy-element enrichment in peculiar stars, it is very crucial to know their binary status. A number of investigations dedicated to identify the binarity of the peculiar stars have been carried out to date. The precise radial velocity monitoring studies have shown that most of the $\mathrm{Ba}$ and $\mathrm{CH}$ stars (McClure et al. 1980, McClure 1983, 1984, McClure \& Woodsworth 1990, Udry et al. 1998a,b, Lucatello et al. 2005, Jorissen et al. 2016) and a high fraction of CEMP-s/(r/s) stars(Lucatello et al. 2005, Starkenburg et al. 2014, Jorissen et al. 2016) are in binary systems. The compilation of Duquennoy \& Mayor (1991) and Starkenburg et al. (2014) have shown the binary fraction of CEMP-s/(r/s) stars to be $100 \%$. However, a few recent studies have reported a binary frequency of $82 \pm 10 \%$ for CEMP-s/(r/s) stars (Hansen et al. 2016c) and $17 \pm 9 \%$ for CEMP-no stars (Hansen et al. 2016b).

All these conclusions are based on the data available to each study. Information available in literature on the binary status of many CEMP stars is still very limited. Yoon et al.(2016) (and references therein) have done a compilation of the literature data for 305 CEMP stars that includes 147 CEMP-s/(r/s) stars and 127 CEMP-no stars. Out of these, 35 CEMP-s/rs stars and 22 CEMP-no stars have known binary status. Earlier to this, Spite et al. (2013) observed for the first time the bimodality in the absolute carbon abundances among the CEMP stars, with CEMP-s stars populating the high-carbon region $(\mathrm{A}(\mathrm{C}) \sim 8.25)$ and CEMP-no stars populating the low-carbon region $(\mathrm{A}(\mathrm{C}) \sim 6.5)$. Later, this bimodality is confirmed by Bonifacio et al (2015) and Hansen et al (2015a) for an extended sample of CEMP stars. Yoon et al. (2016) investigated anew the distribution of absolute carbon abundance, $\mathrm{A}(\mathrm{C})$, in terms of the metallic-

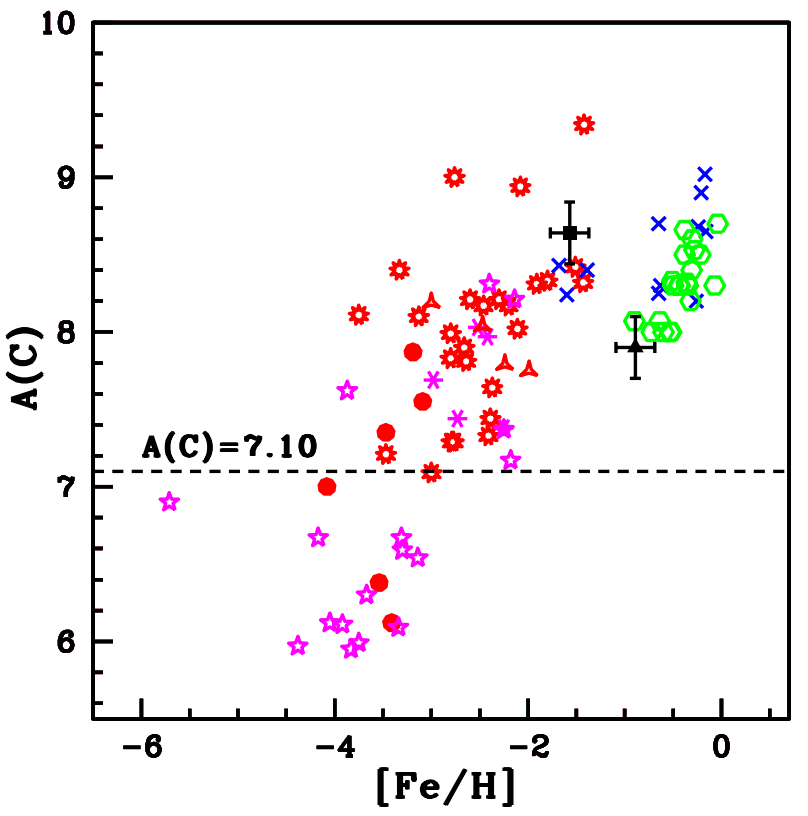

Figure 8. Distribution of $\mathrm{A}(\mathrm{C})$ as a function of $[\mathrm{Fe} / \mathrm{H}]$ for known (and likely) binary and single stars. Red nine-sided stars, magenta six-sided crosses, red starred triangles, red filled circles and magenta five-sided stars represent binary CEMP-s, single CEMP-s, binary CEMP-r/s, binary CEMP-no and single CEMP-no stars respectively from literature (Yoon et al. 2016). All the red symbols corresponds to the binary CEMP stars and magenta symbols to single CEMP stars. Blue crosses represent the binary CH stars from literature (Purandardas et al. 2019, Karinkuzhi \& Goswami 2014, 2015, Luck 2017). Binary Ba stars from literature (Shejeelammal et al. 2020, Karinkuzhi et al. 2018) are represented by green open hexagons. LAMOSTJ091608.81+230734.6 (filled triangle) and LAMOSTJ151003.74+305407.3 (filled square). The dashed line indicate the fiducial that separates binary and single stars.

ity for these CEMP stars. Their analysis have shown that the fiducial line at $\mathrm{A}(\mathrm{C})=7.1$ in the $[\mathrm{Fe} / \mathrm{H}]$ versus $\mathrm{A}(\mathrm{C})$ diagram could well separate the binary nature of all the CEMP$\mathrm{s} /(\mathrm{r} / \mathrm{s})$ stars and majority of CEMP-no stars. Despite of a few outliers, majority of the binary stars lie above this line. Thus this diagram can be used as a tool to derive clues on the binary status of the CEMP stars as well as the origin of their chemical peculiarities. We have used such a figure to understand the binary nature of our program stars. The distribution of absolute carbon abundance with metallicity for different classes of chemically peculiar stars are shown in figure 8 . In this figure, both the program stars lie in the region occupied by binary stars, indicating that our program stars are likely binaries. In addition, the variation in radial velocity estimate for LAMOSTJ151003.74+305407.3 by $\sim 4$ $\mathrm{kms}^{-1}$ also suggests that this object could be a binary star.

\section{DISCUSSION}

From the multiple line indices measured from the stellar spectra from LAMOST DR2, Ji et al. (2016) have identified the objects LAMOSTJ091608.81+230734.6 and LAMOSTJ151003.74+305407.3 
Table 7. Spatial velocity and probability estimates for the program stars

\begin{tabular}{|c|c|c|c|c|c|c|c|}
\hline Star name & $\begin{array}{c}\mathrm{U}_{L S R} \\
\left(\mathrm{kms}^{-1}\right)\end{array}$ & $\begin{array}{c}\mathrm{V}_{L S R} \\
\left(\mathrm{kms}^{-1}\right)\end{array}$ & $\begin{array}{c}\mathrm{W}_{L S R} \\
\left(\mathrm{kms}^{-1}\right)\end{array}$ & $\begin{array}{c}\mathrm{V}_{\text {spa }} \\
\left(\mathrm{kms}^{-1}\right)\end{array}$ & $\mathrm{p}_{\text {thin }}$ & $\mathrm{p}_{\text {thick }}$ & Phalo \\
\hline LAMOSTJ091608.81+230734.6 & $-12.14 \pm 2.99$ & $1.57 \pm 1.41$ & $2.71 \pm 2.95$ & $12.53 \pm 1.98$ & 0.99 & 0.01 & 0.00 \\
\hline LAMOSTJ151003.74+305407.3 & $-16.38 \pm 2.84$ & $-207.80 \pm 20.74$ & $-49.68 \pm 8.67$ & $214.25 \pm 22.33$ & 0.00 & 0.29 & 0.71 \\
\hline
\end{tabular}

to be $\mathrm{CH}$ stars. Our detailed abundance analysis confirms LAMOSTJ091608.81+230734.6 to be a CH star and LAMOSTJ151003.74+305407.3 to be a CEMP -r/s star. The estimated abundances of light elements from $\mathrm{Na}$ to $\mathrm{Zn}$ are quite similar to the normal giants following the Galactic trend as shown in Figure 9. The estimated abundances of heavy elements however show enhancement compared to their counterparts in other normal stars (Figure 10).

The neutron density dependent $[\mathrm{Rb} / \mathrm{Zr}]$ ratio has been estimated in order to see the nature of companion star. For this ratio, the AGB models predict a negative value in low-mass $A G B$ stars $\left(M \leq 3 \mathrm{M}_{\odot}\right)$ and a positive value in intermediate-mass $\mathrm{AGB}$ stars $\left(\mathrm{M} \geq 4 \mathrm{M}_{\odot}\right.$ ) (Abia et al. 2001, van Raai et al. 2012, Karakas et al. 2012). This trend has been observed among the AGB stars in the Galaxy and Magellanic Clouds (Plez et al. 1993, Lambert et al. 1995, Abia et al. 2001, García-Hernández et al. 2006, 2007, 2009). The estimated values of this ratio (Table 8) are shown in Figure 11. The range of $\mathrm{Rb}$ and $\mathrm{Zr}$ observed in the Galactic and Magellanic Cloud low- and intermediate-mass AGB stars (shaded regions) are also shown in the same figure.

LAMOSTJ091608.81+230734.6: The object is found to be metal-poor with a metallicity of -0.89 . Eventhough we could not find its position on HR diagram, its estimated log g value suggests the star to be on RGB (Allen \& Barbuy 2006). The star shows a mild over abundance of carbon with $[\mathrm{C} / \mathrm{Fe}] \sim 0.36$. Similar values of carbon in $\mathrm{CH}$ stars have been reported in literature (Purandardas et al. 2019, Goswami et al. 2016, Karinkuzhi \& Goswami 2015, Vanture 1992). Nitrogen enhancement compared to carbon in the star, along with the low ${ }^{12} \mathrm{C} /{ }^{13} \mathrm{C}$ ratio of $\sim 8.67$, suggest the $\mathrm{CN}$ processing and the First Dredge-Up (FDU) during the giant phase. Further, with its estimated values of mean s-process abundance ratio, $[\mathrm{s} / \mathrm{Fe}] \sim 1.41$, and $\mathrm{C} / \mathrm{O} \sim 1.38$, this star can be included in the $\mathrm{CH}$ giant category. The star shows negative value for the $[\mathrm{Rb} / \mathrm{Zr}]$, which indicates a low-mass companion AGB as expected for the $\mathrm{CH}$ stars (Figure 11). The estimated [hs/ls] ratio is $\sim 0.37$, indicating the over abundance of second-peak s-process elements over the first peak, as normally seen in most of the $\mathrm{CH}$ stars. Our kinematic analysis shows that this star belongs to Galactic thin disc population.

Figure 12 compares the observed abundance ratios $[\mathrm{Ba} / \mathrm{Fe}]$ and $[\mathrm{Eu} / \mathrm{Fe}]$, the representative $\mathrm{s}-$ and $\mathrm{r}$ - process elements respectively, in different classes of chemically peculiar stars. The star LAMOSTJ091608.81+230734.6 falls in the region occupied by other $\mathrm{CH}$ stars, Ba stars and CEMPs stars. These three classes of stars are known to have the same origin of s-process enhancement, pollution from a binary low-mass AGB companion.

LAMOSTJ151003.74+305407.3: This star is found to be metal-poor with a metallicity $[\mathrm{Fe} / \mathrm{H}] \sim-1.57$ and en- hanced in carbon with $[\mathrm{C} / \mathrm{Fe}]>1$. With its estimated value of $[\mathrm{Ba} / \mathrm{Fe}] \sim 1.39$ and $[\mathrm{Ba} / \mathrm{Eu}] \sim 0.25$, along with the enhanced carbon abundance, the star satisfies to be a CEMP-r/s star (Beers \& Christlieb, 2005, Abate et al. 2016). From the position of the star on the HR diagram, it is found to be a giant. The abundances of heavy elements in this star are well within the range observed in other CEMP-r/s stars (Figure 10). In $[\mathrm{C} / \mathrm{N}]$ versus $\log \left({ }^{12} \mathrm{C} /{ }^{13} \mathrm{C}\right)$ space, this star occupies the same region as the CEMP stars (Figure 13).

From the study conducted by Aoki et al.(2007) for 22 metal-poor stars $(-3.3 \leq[\mathrm{Fe} / \mathrm{H}] \leq-1.0)$ exhibiting strong $\mathrm{CH}$ and/or $\mathrm{C}_{2}$ molecular band, they came up with a new empirical definition for CEMP stars in terms of $[\mathrm{C} / \mathrm{Fe}]$ and $\log \left(\mathrm{L} / \mathrm{L}_{\odot}\right)$. Based on this definition, there exist a clear division between the CEMP stars and the carbon-normal metal-poor stars in the $\log \left(\mathrm{L} / \mathrm{L}_{\odot}\right)$ versus $[\mathrm{C} / \mathrm{Fe}]$ plane. We have demonstrated this in Figure 14. The star LAMOSTJ151003.74+305407.3 is also shown in the same figure. This star falls in the region occupied by the CEMP stars.

The Figure 12 shows the position of the star LAMOSTJ151003.74+305407.3 in the $[\mathrm{Eu} / \mathrm{Fe}]-[\mathrm{Ba} / \mathrm{Fe}]$ space. The star is not placed in the bulk of the CEMP-r/s stars, however, there are a few CEMP-r/s stars in the same region as LAMOSTJ151003.74+305407.3.

With the knowledge that r-process and s-process are ascribed to different astrophysical sites (Burbidge et al. 1957), several formation scenarios for CEMP-r/s stars have been proposed in literature (Cohen et al. 2003, Qian \& Wasserburg 2003, Zijlstra 2004, Barbuy et al. 2005, Wanajo et al. 2006, Jonsell et al. 2006, Bisterzo et al. 2011 and references therein), most of them suggesting different independent processes for the $\mathrm{r}$ - and s- peculiarity. One scenario describes that the CEMP-r/s star could have been a secondary in a binary system formed out of r-process enriched ISM and its s-elements and carbon enhancement is due to the later pollution from the AGB companion through the mass-transfer mechanism (Hill et al. 2000, Cohen et al. 2003, Ivans et al. 2005). But this could not successfully explain the observed frequency of CEMP-r/s stars. The study by Barklem et al. (2005) have revealed that an order of $1 \%$ of population II stars are CEMP-r/s stars. Cohen et al (2003) have discussed another scenario that invokes a triple system in which the star could have been a least massive tertiary, polluted first with the r-elements from the massive primary exploded as supernova and later polluted with s-elements by the secondary star evolved into an AGB. This hypothesis had been discarded, as such a dynamically stable tertiary system is unlikely to exist. Accretion Induced Collapse (AIC) in a binary system have been suggested by Qian \& Wasserburg (2003) and Cohen et al. (2003), but discarded since it is physically uncertain with the existing neutrino theories (Qian \& Woosley 1996). Another scenario is a binary picture in which the primary star evolved through AGB contributed 
Table 8. Estimates of [ls/Fe], [hs/Fe], [s/Fe], [hs/ls], [Rb/Zr], [Ba/Eu] and C/O

\begin{tabular}{lcccccccc}
\hline Star name & {$[\mathrm{Fe} / \mathrm{H}]$} & {$[\mathrm{ls} / \mathrm{Fe}]$} & {$[\mathrm{hs} / \mathrm{Fe}]$} & {$[\mathrm{s} / \mathrm{Fe}]$} & {$[\mathrm{hs} / \mathrm{ls}]$} & {$[\mathrm{Rb} / \mathrm{Zr}]$} & {$[\mathrm{Ba} / \mathrm{Eu}]$} & $\mathrm{C} / \mathrm{O}$ \\
\hline LAMOSTJ091608.81+230734.6 & -0.89 & 1.20 & 1.57 & 1.41 & 0.37 & -0.92 & 0.49 & 1.38 \\
LAMOSTJ151003.74+305407.3 & -1.57 & 1.30 & 1.44 & 1.67 & 0.14 & -0.99 & 0.25 & - \\
\hline
\end{tabular}
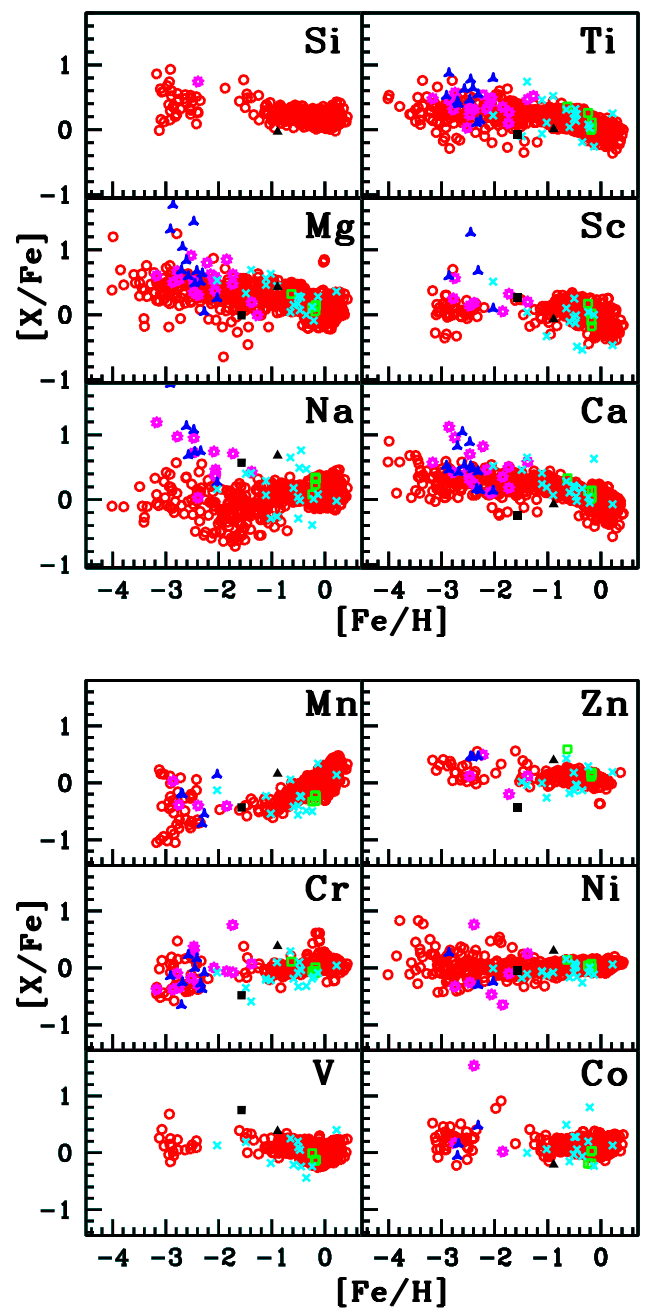

Figure 9. Observed $[\mathrm{X} / \mathrm{Fe}]$ ratios of the light elements in the program stars with respect to metallicity $[\mathrm{Fe} / \mathrm{H}]$. Red open circles correspond to normal giants from literature (Honda et al. 2004, Venn et al. 2004, Aoki et al. 2005, 2007, Reddy et al. 2006, Luck \& Heiter 2007, Hansen et al. 2016a, Yoon et al. 2016). Magenta nine-sided stars and blue starred triangles represent CEMP-s and CEMP-r/s stars respectively from literature (Masseron et al. 2010). Cyan crosses and green open squares represent giant and sub-giant $\mathrm{CH}$ stars respectively from literature (Vanture 1992, Karinkuzhi \& Goswami 2014, 2015, Goswami et al. 2016). LAMOSTJ091608.81+230734.6 (filled triangle) and LAMOSTJ151003.74+305407.3 (filled square).

the s-rich material to the secondary star, and later explodes as Type 1.5 supernova (Zijlstra 2004, Wanajo et al. 2005) depositing r-material on the surface of the secondary. Abate et al. (2016) have calculated the frequency of CEMP-r/s stars among the CEMP-s stars for all these formation scenarios. The theoretical frequency predicted in most of the scenar-
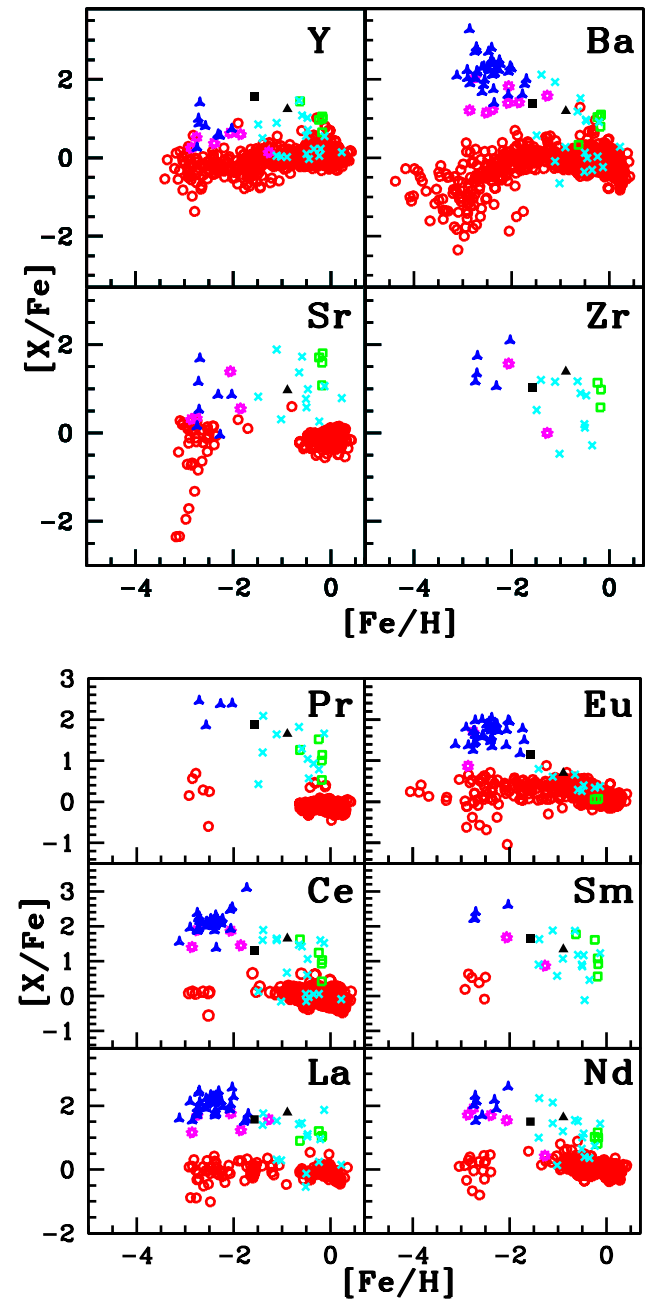

Figure 10. Observed $[\mathrm{X} / \mathrm{Fe}]$ ratios of the heavy elements in the program stars with respect to metallicity $[\mathrm{Fe} / \mathrm{H}]$. Symbols have same meaning as in Figure 9.

ios underestimate the observed frequency $(\sim 54 \%$ in their sample) atleast by a factor of five. The simulation based on the hypothesis of independent enrichment of s- and r-elements could predict a frequency $(\sim 22 \%)$ that approaches the observations, however, it fails to reproduce the observed correlation of observed $\mathrm{Ba}$ and $\mathrm{Eu}$ abundances in CEMP$\mathrm{r} / \mathrm{s}$ stars. The simulations of Jonsell et al. (2006) for a high neutron density s-process in AGB star in a binary system also could not reproduce the observed abundance pattern in CEMP-r/s stars.

Allen et al. (2012) have claimed from their analysis for a sample of CEMP stars that both CEMP-s and CEMP-r/s stars have same astrophysical origin. A modified neutroncapture process called intermediate neutron-capture process 


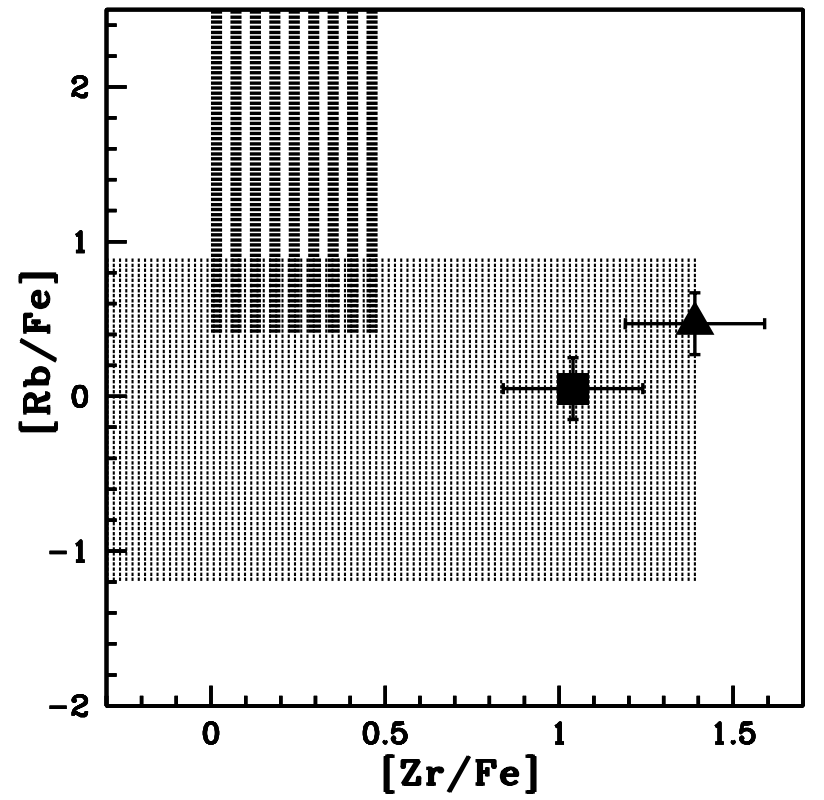

Figure

11.

The

observed

$[\mathrm{Rb} / \mathrm{Fe}]$ and $[\mathrm{Zr} / \mathrm{Fe}]$. LAMOSTJ091608.81+230734.6 (filled triangle) and LAMOSTJ151003.74+305407.3 (filled square). The shaded regions represent the observed ranges of $\mathrm{Zr}$ and $\mathrm{Rb}$ in intermediate-mass (short-dashed lines) and low-mass (dots) AGB stars of the Galaxy and the Magellanic Clouds (van Raai et al. 2012). The $\mathrm{Rb}$ and $\mathrm{Zr}$ abundances in the program stars are consistent with that of low-mass AGB stars.

(i-process) in AGB stars, first proposed by Cowan \& Rose (1977), has been invoked recently (Dardelet et al. 2014, Hampel et al 2016, 2019, Hansen et al. 2016c) to explain the observed abundance trend of CEMP-r/s stars in the context of binary-mass transfer scenario. When a substantial amount of hydrogen rich-material is mixed into the intershell region of the evolved red-giant stars (Proton Ingestion Episodes, PIE) undergoing helium shell flash, a significantly high neutron density of the order $\mathrm{N}_{n} \sim 10^{15}-10^{17} \mathrm{~cm}^{-3}$ (intermediate between s- and r- processes) can be produced (Cowan \& Rose 1977). A number of sites have been proposed for the PIEs in order for the i-process nuclesynthesis to take place. Recent simulations have shown that the neutron-densities of the order of $10^{12}-10^{15} \mathrm{~cm}^{-3}$ can be achieved in very lowmetallicity $\left(\mathrm{z} \leq 10^{-4}\right)$, low-mass $\left(\mathrm{M} \leq 2 \mathrm{M}_{\odot}\right)$ AGB stars (Fujimoto et al. 2000, Campell \& Lattanzio 2008, Lau et al. 2009, Cristallo et al. 2009, Campbell et al. 2010, Stancliffe et al. 2011). A similar neutron density is achieved during the dual core flash $(\mathrm{H}$ flash following the PIEs during the He flash) in low-mass, extremely low-metallicity $\left(\mathrm{z} \leq 10^{-5}\right)$ stars (Fujimoto et al. 1990, Hollowell et al. 1990, Lugaro et al. 2009); in the very-late thermal pulses of post-AGB stars (Herwig et al. 2011, 2014 Bertolli et al. 2013, Woodward et al. 2015); during the thermal pulses of low-metallicity super-AGB stars (Doherty et al. 2015, Jones et al. 2016); in Rapidly Accreting White Dwarfs (RAWD) (Denissenkov et al. 2019). Dardelet et al. (2014), Hampel et al. (2016) and Hampel et al. (2019) considered the possibility of i-process in their simulation to see whether the CEMP-r/s phenomena could be explained on the basis of s- and $r-$ process el-

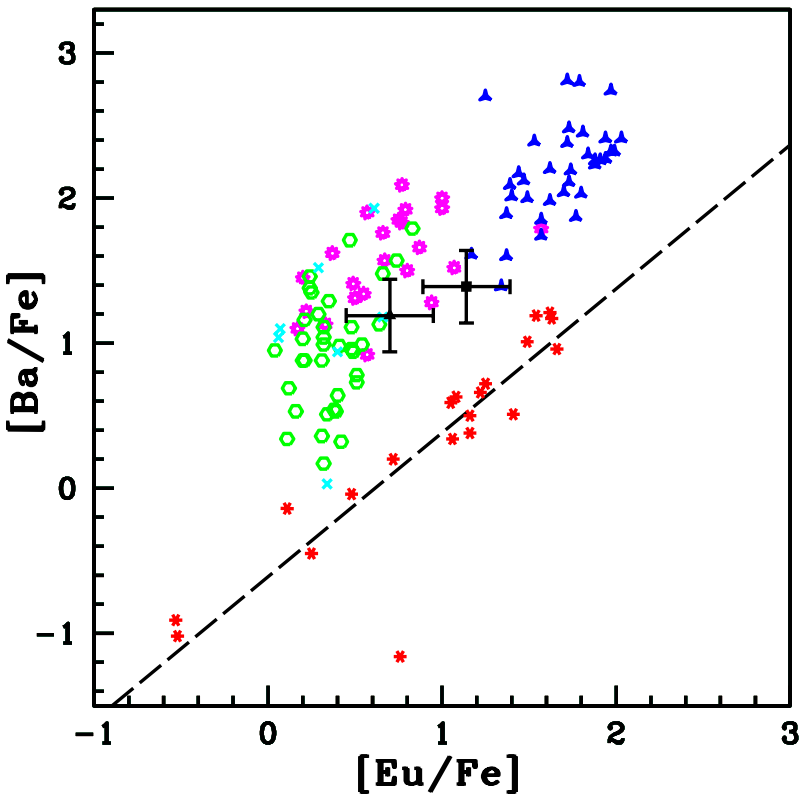

Figure 12. Observed $[\mathrm{Eu} / \mathrm{Fe}]$ and $[\mathrm{Ba} / \mathrm{Fe}]$ ratios for different classes of chemically peculiar stars. Magenta nine-sided stars, blue starred triangles and red six-sided crosses represent CEMP-s CEMP-r/s and $r$ (including both CEMP-r and $\mathrm{rI} / \mathrm{rII}$ stars) stars respectively from literature (Masseron et al. 2010). Cyan crosses represent $\mathrm{CH}$ stars from literature (Vanture 1992, Karinkuzhi \& Goswami 2014, 2015, Goswami et al. 2016). Green open hexagons represent the Ba stars from literature (Shejeelammal et al. 2020, Yang et al. 2016, Allen \& Barbuy 2006). LAMOSTJ091608.81+230734.6 (filled triangle) and LAMOSTJ151003.74+305407.3 (filled square). The dashed line is the least-square fit to the observed abundances in r-stars.

ements produced at a single stellar site. The i-process models could satisfactorily reproduce the abundance patterns of twenty CEMP-r/s stars (Hampel et al. 2016) and seven low$\mathrm{Pb}$ Magellanic post-AGB stars (Hampel et al. 2019).

Hampel et al. (2016) used the single-zone nuclear network calculations to simulate the properties of the intershell region of low-mass $\left(1 \mathrm{M}_{\odot}\right)$, low-metallicity $\left(\mathrm{z}=10^{-4}\right) \mathrm{AGB}$ star and studied the neutron-capture nucleosynthesis under the influence of different constant neutron densities ranging from $10^{7}$ to $10^{15} \mathrm{~cm}^{-3}$. The physical input conditions of intershell region are adapted from Stancliffe et al. (2011) and the composition from Abate et al. (2015b). The considered temperature and density of the intershell region are $1.5 \times$ $10^{8} \mathrm{~K}$ and $1600 \mathrm{~g} \mathrm{~cm}^{-3}$ respectively. Compared to the classical s-process, at i-process neutron densities, this simulation resulted in an increased production of heavy s-process and rprocess elements, while similar abundances of light s-process elements, as typically observed for CEMP-r/s stars (Abate et al. 2015a, Hollek et al. 2015). A range of temperatures $\left(1.0 \times 10^{8}-2.2 \times 10^{8} \mathrm{~K}\right)$ and densities $\left(800-3200 \mathrm{~g} \mathrm{~cm}^{-3}\right)$ have been tested in this simulation, which did not produce significant changes in the result.

We have compared the observed heavy elemental abundance ratios of the star LAMOSTJ151003.74+305407.3 with the model yields, [X/Fe], of Hampel et al. (2016) for a range of neutron densities, $\mathrm{n} \sim 10^{9}-10^{15} \mathrm{~cm}^{-3}$. A further dilution of the accreted material can occur on the surface of the 


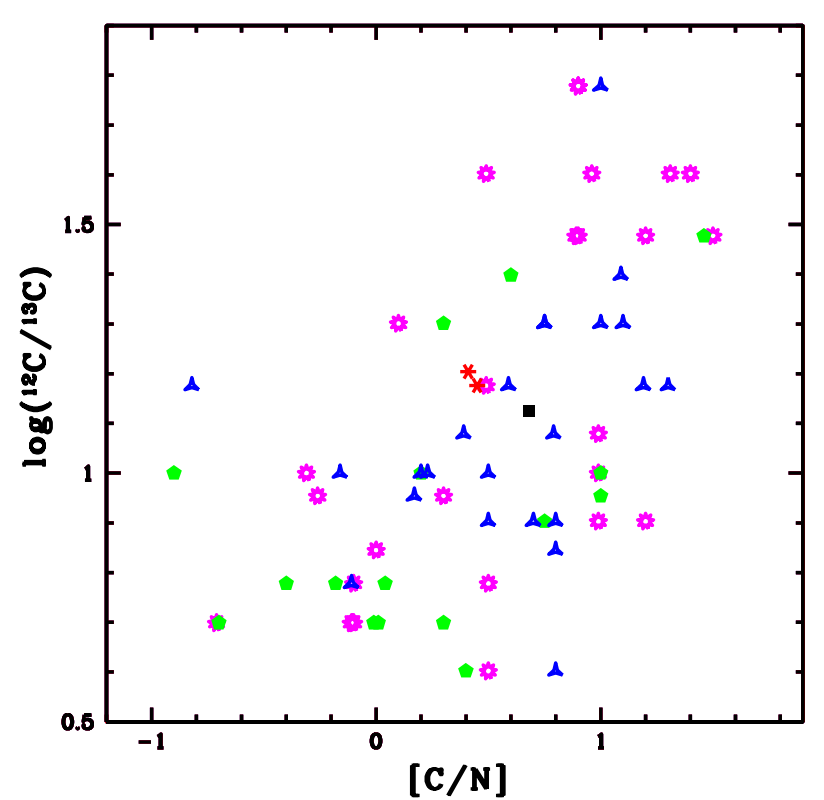

Figure 13. Observed $[\mathrm{C} / \mathrm{N}]$ and $\log \left({ }^{12} \mathrm{C} /{ }^{13} \mathrm{C}\right)$ ratios of the CEMP stars. Magenta nine-sided stars, red six-sided crosses, blue starred triangles and green filled pentagons represent CEMP-s, CEMP-r, CEMP-r/s and CEMP-no stars respectively from literature (Masseron et al. 2010). LAMOSTJ151003.74+305407.3 (filled square).

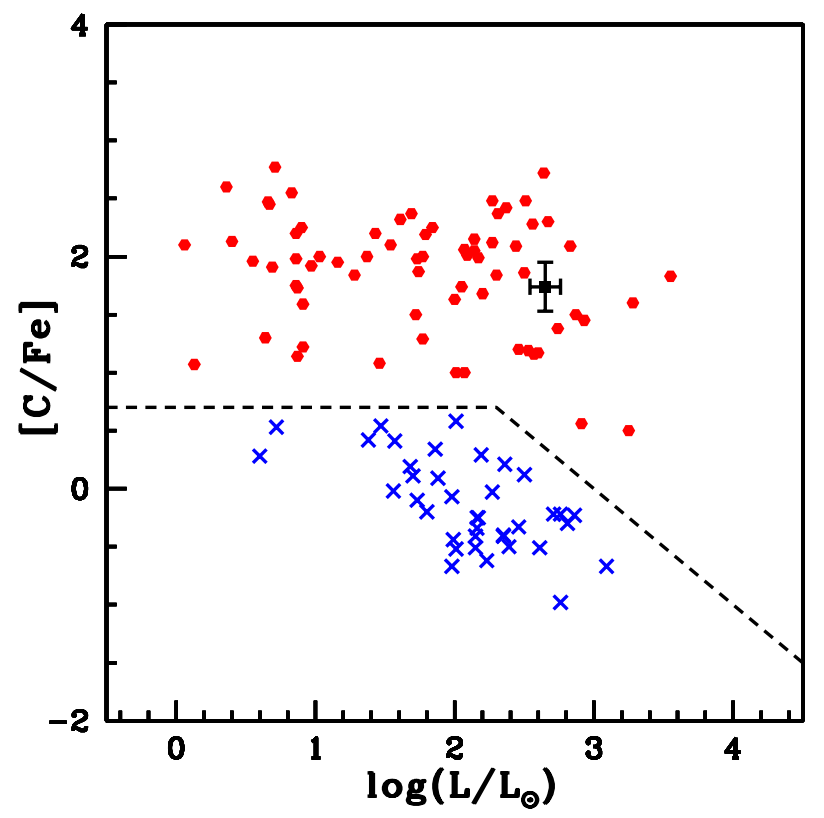

Figure 14. Observed $[\mathrm{C} / \mathrm{Fe}]$ ratios as a function of luminosity estimated from the effective temperature. Red filled hexagons represent CEMP stars from literature (Aoki et al. 2007 and references therein, Purandardas et al. 2019, Goswami et al. 2016). Blue crosses represent carbon-normal metal-poor stars from literature (Aoki et al. 2005, 2007, Cayrel et al. 2004, Honda et al. 2004). LAMOSTJ151003.74+305407.3 (filled square). The dashed line indicates the dividing line between CEMP and the carbonnormal metal-poor stars.

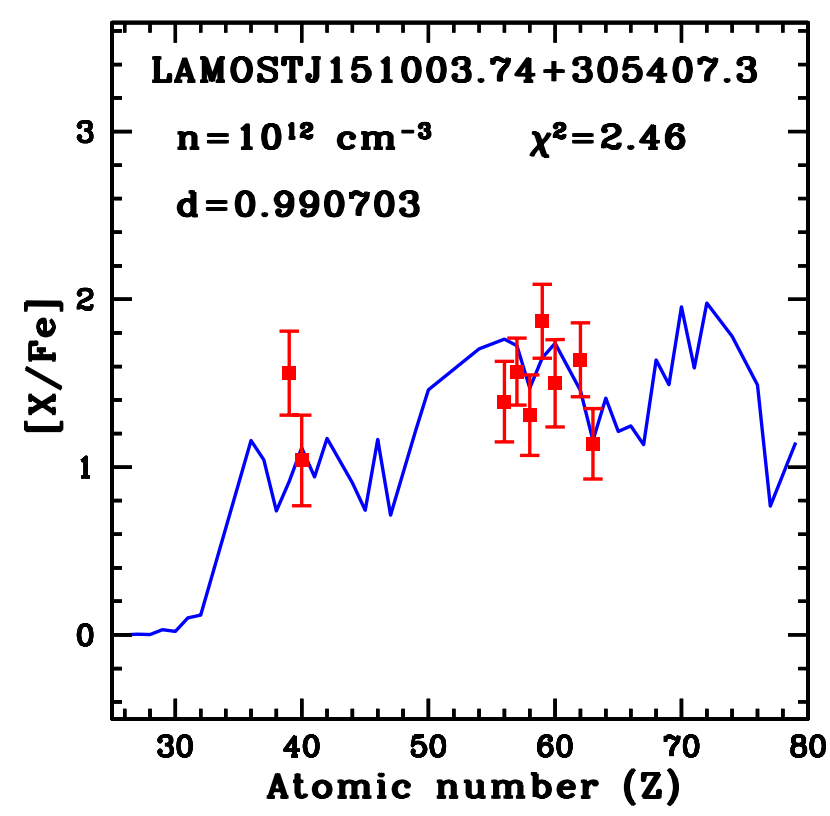

Figure 15. The best fit obtained for the parametric model function is represented by the solid line. The squares with error bars are the observed abundances in LAMOSTJ151003.74+305407.3.

CEMP-r/s stars just like similarly formed CEMP-s stars (Stancliffe et al. 2007, 2013). The neutron-density responsible for the observed abundance in this star is derived by fitting the observed abundance with the dilution factor incorporated parametric model function:

$\mathrm{X}=\mathrm{X}_{i} \cdot(1-\mathrm{d})+\mathrm{X} \odot \cdot \mathrm{d}$

where $\mathrm{X}$ is the final abundance, $\mathrm{X}_{i}$ is the $\mathrm{i}$-process abundance, $\mathrm{X}_{\odot}$ is the solar-scaled abundance and $\mathrm{d}$ is the dilution factor. The best fit obtained along with the neutron density and dilution factor is shown in Figure 15. The neutron density responsible for the observed abundance of this star is found to be $\mathrm{n} \sim 10^{12} \mathrm{~cm}^{-3}$, if we assume a single stellar site for the production of the observed neutron-capture abundance pattern.

From the kinematic analysis, we find that this object belongs to the Galactic halo with a probability of $71 \%$. The spatial velocity estimate of the star is similar to the typical velocity of halo objects, $\mathrm{V}_{\text {spa }}>180 \mathrm{kms}^{-1}$ (Chen et al. 2004). Also it satisfies the criteria, $[\mathrm{Fe} / \mathrm{H}]<-0.90$ and $\mathrm{V}_{L S R}<-120$ $\mathrm{km} \mathrm{s}^{-1}$ (Eggen 1997) to be a halo object.

\section{CONCLUSIONS}

The results from a detailed high-resolution spectroscopic analysis of two carbon stars identified from LAMOST DR2 are presented. Both the objects are identified to be $\mathrm{CH}$ stars by Ji et al. (2016). Our analysis shows that the object LAMOSTJ151003.74+305407.3 is a CEMP-r/s star, while the object LAMOSTJ091608.81+230734.6 is a $\mathrm{CH}$ star.

Although, a few light element abundances are available in literature for LAMOSTJ151003.74+305407.3, we have presented the first time detailed abundance analysis for both 
the stars. We have estimated the abundances of 26 elements along with the carbon isotopic ratio ${ }^{12} \mathrm{C} /{ }^{13} \mathrm{C}$.

Our analysis based on the neutron-density dependent $[\mathrm{Rb} / \mathrm{Zr}]$ ratio confirms low-mass for the former AGB companion of the program stars. The kinematic analysis shows, LAMOSTJ091608.81+230734.6 belongs to the Galactic disc, and LAMOSTJ151003.74+305407.3 belongs to Galactic halo population.

An i-process

parametric model based analysis performed for the CEMP-r/s star LAMOSTJ151003.74+305407.3, yields a neutron density $\mathrm{n} \sim 10^{12} \mathrm{~cm}^{-3}$ at the neutron-capture nucleosynthesis site, may indicate that the i-process in the companion AGB star be responsible for its observed abundance pattern.

\section{ACKNOWLEDGMENT}

We thank the staff at IAO and at the remote control station at CREST, Hosakotte for assisting during the observations. Funding from the DST SERB project No. EMR/2016/005283 is gratefully acknowledged. We are thankful to the referee, Dr. Luca Sbordone, for useful comments and suggestions that have considerably improved the paper. We are thankful to Melanie Hampel for providing us with the i-process yields in the form of number fractions and Partha Pratim Goswami for generating the model fits used in Figure 15. This work made use of the SIMBAD astronomical database, operated at CDS, Strasbourg, France, and the NASA ADS, USA. This work has made use of data from the European Space Agency (ESA) mission Gaia (https://www.cosmos.esa.int/gaia), processed by the Gaia Data Processing and Analysis Consortium (DPAC, https://www.cosmos.esa.int/web/gaia/dpac/consortium).

Based on data collected using HESP

\section{Data Availability}

The data underlying this article will be shared on reasonable request to the corresponding author.

\section{REFERENCES}

Abate C., Pols O. R., Izzard R. G. \& Karakas A. I., 2015a, A\&A, 581, A22

Abate C., Pols O. R., Karakas A. I. \& Izzard R. G., 2015b, A\&A, 576, A118

Abate C., Stancliffe R.J., Liu Z-W., 2016, A\&A, 587, 50

Abia C., Busso M., Gallino R., Domínguez I., Straniero O. \& Isern J., 2001, ApJ, 559, 1117

Alksnis A., Balklavs A., Dzervitis U. et al., 2001, BaltA, 10, 1

Allen D.M., Barbuy B., 2006, A\&A, 454, 895

Allen D. M., Ryan S. G., Rossi S., Beers T. C. \& Tsangarides S. A., 2012, A\&A, 548, A34

Alonso A., Arribas S., Martinez-Roger C., 1999, A\&AS, 140, 261

Alonso A., Arribas S., Martinez-Roger C., 2001, A\&A, 376, 1039

Aoki W. et al., 2005, ApJ, 632, 611

Aoki W., Beers T.C., Christlieb N., Norris J.E., Ryan S.G et al., 2007, ApJ, 655, 492

Aoki W. et al., 2013, AJ, 145, 13

Asplund M., Grevesse N., Sauval A.J., 2009, Ann. Rev. Astron. Astrophy., 47, 481

Banerjee P., Qian Y.-Z. \& Heger A., 2018, ApJ, 865, 120
Barbuy B., Spite M., Spite F., Hill V., Cayrel R., Plez B., Petitjean P., 2005, A\&A, 429, 1031

Barklem P. S., Christlieb N., Beers T. C. et al., 2005, A\&A, 439, 129

Beers, T. C. 1999, in ASP Conf. Ser. 165, The Third Stromlo Symposium: The 1443 Galactic Halo, ed. B. Gibson, T. Axelrod, \& M. Putman (San Francisco: Hill, V., et al. 2000, A\&A, 353, 557 ASP), 202

Beers T. C., Preston G. W. \& Shectman S. A., 1985, AJ, 90, 2089

Beers T. C., Preston G. W. \& Shectman S. A., 1992, AJ, 103, 1987

Beers T. C., Flynn C., Rossi S. et al., 2007, ApJS, 168, 128

Beers T. C., Christlieb N., 2005, ARA\&A, 43, 531

Bensby T., Feltzing S., Lundstrom I., 2003, A\&A, 410, 527

Bensby T., Feltzing S., Lundstrom I., 2004, A\&A, 415, 155

Bertolli M. G., Herwig F., Pignatari M. \& Kawano T., 2013, ArXiv e-prints [arXiv:1310.4578]

Biemont E., Grevesse N., Hannaford P., Lowe R.M., 1981, ApJ 248, 867-873

Bisterzo S., Gallino R., Straniero O., Cristallo S., Käppeler F., 2011, MNRAS, 418, 284

Bonifacio P., Caffau E., Spite M., Limongi M., Klessen R. S. et al., 2015, A\&A, 579, A28

Brooke J. S. A., Bernath P. F., Schmidt T. W. \& Bacskay G. B., 2013, JQSRT, 124, 11

Burbidge E. M., Burbidge G. R., Fowler W. A. \& Hoyle F., 1957, RvMP, 29, 547

Campbell S. W. \& Lattanzio J. C., 2008, A\&A, 490, 769

Campbell S. W., Lugaro M. \& Karakas A. I., 2010, A\&A, 522, L6

Carollo D. et al., 2012, ApJ, 744, 195

Cayrel R. et al., 2004, A\&A, 416, 1117

Chen Y.Q., Nissen P.E., Zhao G., 2004, A\&A, 425, 697

Christlieb N., 2003, RvMA, 16, 191

Christlieb N., Green P. J., Wisotzki L., \& Reimers D., 2001a, A\&A, 375, 366

Christlieb N., Wisotzki L., Reimers D., Homeier D., Koester D. \& Heber U., 2001b, A\&A, 366, 898

Clarkson O., Herwig F. \& Pignatari M., 2018, MNRAS, 474, L37

Cohen J.G., Christlieb N., Qian Y.Z., Wasserburg G.J., 2003, AJ, 588, 1082

Cohen J. G. et al., 2005, ApJ, 633, L109

Corliss C.H., Bozman W.R., 1962, NBS Monograph 53

Cowa J. J. \& Rose W. K., 1977, ApJ, 212, 149

Cowley C.R., Corliss C.H., 1983, MNRAS 203, 651-659

Cristallo S., Straniero O., Gallino R., Piersanti L., Domínguez I., Lederer M. T., 2009, ApJ, 696, 797

Cristallo S., Karinkuzhi D., Goswami A., Piersanti L. \& Gobrecht D., 2016, ApJ, 833, 181

Cruz M. A., Serenelli A. \& Weiss A., 2013, A\&A, 559, A4

Cui X., Zhao Y. H., Chu Y. Q. et al., 2012, RAA, 12, 1197

Dardelet L., Ritter C., Prado P. et al., 2014, in POS XIII Nuclei in the Cosmos (NIC XIII), ed. E. Zoltán \& Z. Fülöp, 145

Deng L., Newberg H. J., Liu C. et al., 2012, RAA, 12, 735

Denissenkov P. A., Herwig F., Battino U. et al., 2017, ApJL, 834, L10

Denissenkov P. A., Herwig F., Woodward P. et al., 2019, MNRAS, 488, 4258

Doherty C. L., Gil-Pons P., Siess L., Lattanzio J. C. \& Lau H. H. B., 2015, MNRAS, 446, 2599

Duquennoy A. \& Mayor M., 1991, A\&A, 248, 485

Eggen O.J., 1997, AJ, 114, 825

Frebel A., 2018, Annual Review of Nuclear and Particle Science, 68,237

Frebel A. \& Norris J. E., 2015, ARA\&A, 53, 631

Frebel A. et al., 2006, ApJ, 652, 1585

Führ J.R., Martin G.A., Wiese W.L., 1988, J. Phys. Chem. Ref. Data 17 , Suppl. 4

Fujimoto M. Y., Iben I. Jr. \& Hollowell D., 1990, ApJ, 349, 580 
Fujimoto M. Y., Ikeda Y. \& Iben I. Jr., 2000, ApJL, 529, L25

Gaia collaboration, Kartz D., Antoja T. et al., 2018, A\&A, 616, A11

García-Herández D. A., García-Lario P., Plez B., D'Antona F., Manchado A., Trigo-Rodríguez M., 2006, Science, 314, 1751

García-Herández D. A., García-Lario P., Plez B., Manchado A., D'Antona F., Lub J. \& Habing H., 2007, A\&A, 462, 711

García-Herández D. A. et al., 2009, ApJ, 705, L31

Garz T., 1973, A\&A. 26, 471.

Gigoyan K. S., HamBaryan V. V. \& Azzopardi M., 1998, APADS, 41,545

Girardi L., Bressan A., Bertelli G., Chiosi C., 2000, A\&AS 141, 371

Goswami A., Aoki W., Karinkuzhi D., 2016, MNRAS, 455, 402

Hampel M., Stancliffe R.J., Lugaro M., Meyer B.S., 2016, ApJ, 831,171

Hampel M., Karakas A. I., Stancliffe R. J., Meyer B. S., Lugaro M., 2019, ApJ, 887, 11

Hannaford P., Lowe R.M., Grevesse N., Biemont E., Whaling W., 1982, ApJ 261, 736-746

Hansen T., Hansen C. J., Christlieb N., Beers T. C., Yong D. et al., 2015a, ApJ, 807, 173

Hansen C. J., Nordström B., Hansen T. T., Kennedy C. R., Placco V. M. et al., 2016a, A\&A, 588, A37

Hansen T. T., Andersen J., Nordström B., Beers T. C., Placco V. M. et al., 2016b, A\&A, 586, A160

Hansen T. T., Andersen J., Nordström B., Beers T. C., Placco V. M. et al., 2016c, A\&A, 588, A3

Hansen C. J., Hansen T. T., Koch A. et al., 2019, A\&A, 623, A128

Hayes C. R., Majewski S. R., Shetrone M., Fernandez-Alvar E., Prieto C. A. et al., 2018, ApJ, 852, 49H

Herwig F., Pignatari M., Woodward P. R. et al., 2011, ApJ, 727, 89

Herwig F., Woodward P. R., Lin P.-H., Knox M. \& Fryer C., 2014, ApJL, 792, L3

Hill V., Barbuy B., Spite M. et al., 2000, A\&A, 353, 557

Hollek J. K., Frebel A., Placco V. M. et al., 2015, ApJ, 814, 121

Hollowell D., Iben I. Jr. \& Fujimoto M. Y., 1990, ApJ, 351, 245

Honda S., Aoki W., Kajino T., Ando H., Beers T. C. et al., 2004, ApJ, 607, 474

Ibata R., Lewis G. F., Irwin M., Totten E. \& Quinn T., 2001, ApJ, 551, 294

Ivans I. I., Sneden C., Gallino R. et al., 2005, ApJ, 627, L145

Ji W., Cui W., Liu C., Luo A., Zhao G. \& Zhao B., 2016, ApJS, 226,1

Johnson D.R.H., Soderblom D.R., 1987, AJ, 93, 864

Jones S., Ritter C., Herwig F. et al., 2016, MNRAS, 455, 3848

Jonsell K., Barklem P.S., Gustafsson B., Christlieb N., Hill V. et al., 2006, A\&A, 451, 651

Jorissen A., Eck S. V., Winckel H. V., Merle T., Boffin H. M. J. et al., 2016, A\&A, 586, 158

J. Shejeelammal., Goswami A., Goswami P. P., Rathour R. S., Masseron T., 2020, MNRAS, 492, 3708

Käppeler F., Gallino R., Bisterzo S., Aoki W., 2011, RvMP, 83, 157

Karakas A.I., García-Hernández D. A. \& Lugaro M., 2012, ApJ, 751,8

Karinkuzhi D., Goswami A., 2014, MNRAS, 440, 1095

Karinkuzhi D., Goswami A., 2015, MNRAS, 446, 2348

Karinkuzhi D., Van Eck S., Jorissen A., Goriely S., Siess L., Merle T., Escorza A., Van der Swaelmen M., Boffin H. M. J., Masseron T., Shetye S. \& Plez B., 2018, A\&A, 618, A32

Keenan P.C., 1942, ApJ, 96, 101

Koch A., Reichert M., Hansen C. J. et al., 2019, A\&A, 622, A159

Kurucz R.L., Peytremann E., 1975, SAO Special Report 362

Kurucz R.L., 1988, Trans. IAU, XXB, M. McNally, ed., Dordrecht: Kluwer, 168-172
Lage C.S., Whaling W., 1976, JQSRT 16, 537-542

Lambert D.L., Mallia E.A. \& Warner B., 1969, MNRAS 142, 71.

Lambert D. L. \& Luck R. E., 1976, Obs., 96, 100L

Lambert D. L., Smith V. V., Busso M., Gallino R. \& Straniero O., 1995, ApJ, 450, 302

Lambert D.L., Heath J.E., Lemke M., Drake J., 1996, ApJS, 103, 183

Lau H. H. B., Stancliffe R. J. \& Tout C. A., 2009, MNRAS, 396, 1046

Lee Y. S. et al., 2013, AJ, 146, 132

Lincke R., Ziegenbein G., 1971, Z. Phyzik, 241, 369.

Lucatello S., Tsangarides S., Beers T. C., Carretta E., Gratton R. G., Ryan S. G., 2005, ApJ, 652, 825

Lucatello S., Beers T. C., Christlieb N., Barklem P. S., Rossi S. et al., 2006, ApJ, 652, L37

Luck R. E., 2017, AJ, 153, 21

Luck R. E. \& Heiter U., 2007, AJ, 133, 2464

Lugaro M., Campbell S. W. \& de Mink S. E., 2009, PASA, 26, 322

Marsteller B., Beers T. C., Rossi S., Christlieb N., Bessell M., Rhee J., 2005, Nucl. Phys. A, 758, 312

Martin G.A., Führ J.R., Wiese W.L., 1988, J.Phys.Chem.Ref.Data, 17, Suppl.3

Masseron T., Johnson J.A., Plez B., Van Eck S., Goriely S., Jorissen A., 2010, A\&A, 509, 93

McClure R.D., 1983, ApJ, 208, 264

McClure R.D., 1984, ApJ, 280, 31

McClure R.D., Woodsworth W., 1990, ApJ, 352, 709

McClure R. D., Fletcher J. M., Nemec J., 1980, ApJ, 238, L35

McWilliiam A., 1998, AJ, 115, 1640

Meggers W.F., Corliss C.H., Scribner B.F., 1975, NBS Monograph 145

Mishenina T.V., Soubiran C., Kovtyukh V.V., Korotin S.A., 2004, A\&A, 418, 551

Norris J. E., Ryan S. G., Beers T. C., 1997, ApJ, 488, 350

Placco V. M., Frebel A., Beers T. C. \& Stancliffe R. J., 2014, ApJ, 797, 21

Plez B., Smith V. V. \& Lambert D. L., 1993, ApJ, 418, 812

Prochaska J.X., McWilliam A., 2000, ApJ, 537, 57

Prochaska J. X., Naumov S. O., Carney B. W., McWilliam A., Wolfe A. M., 2000, AJ, 120, 2513

Purandardas M., Goswami A., Goswami P. P., Shejeelammal J., Masseron T., 2019, MNRAS, 486, 3266

Qian Y.-Z \& Wasserburg G. J., 2003, ApJ, 588, 1099

Qian Y.-Z. \& Woosley, 1996, ApJ, 471, 331

Ram R. S., Brooke James S. A., Bernath P. F., Sneden C., Lucatello S., 2014, ApJS, 211, 5

Reddy B.E., Lambert D.L., Priesto C.A., 2006, MNRAS, 367, 1329

Rossi S., Beers T. C., Sneden C., 1999, in Gibson B. K., Axelrod R. S., Putman M. E., eds, ASP Conf. Ser., Vol. 165, The Third Stromlo Symposium: The Galactic Halo. Astron. Soc. Pac., San Francisco, p. 264

Rossi S., Beers T. C., Sneden C. et al., 2005, AJ, 130, 2804

Schönrich R., Binney J., Dehnen W., MNRAS, 2010, 403, 1829

Schulz-Gulde E., 1969, JQSRT 9, 13

Smith P. L., Kuhne M., 1978, Proc. Roy. Soc., 363, 263

Smith V. V., Coleman H. \& Lambert D. L., 1993, ApJ, 417, 287

Sneden C., 1973, PhD thesis, Univ. Texas

Sneden C., Cowan J.J., Gallino R., 2008, ARA\&A, 46, 241

Sneden C., Lucatello S., Ram R. S., Brooke J. S. A. \& Bernath P. F., 2014, ApJS, 214, 26

Spite M., Caffau E., Bonifacio P., Spite F., Ludwig H. -G. et al., 2013, A\&A, 552, A107

Stancliffe R.J., Glebbeek E., Izzard R.G., Pols O.R., 2007, A\&A, 464,57

Stancliffe R. J., Dearborn D. S. P., Lattanzio J. C., Heap S. A. \& Campbell S. W., 2011, ApJ, 742, 121 
Stancliffe R. J., Kennedy C. R., Lau H. H. B. \& Beers T. C., 2013, MNRAS, 435, 698

Starkenburg E., Shetrone M D., McConnachie A W., Venn K A., 2014, MNRAS, 441, 1217

Totten E. J. \& Irwin M. J., 1998, MNRAS, 294, 1

Udry S., Jorissen A., Mayor M., Van Eck S., 1998a, A\&AS, 131, 25

Udry S., Mayor M., Van Eck S., Jorissen A., Prévot L., Grenier S., Lindgren H., 1998b, A\&AS, 131, 43

van Raai M. A., Lugaro M., Karakas A. I., García-Hernndez D. A. \& Yong D., 2012, A\&A, 540, A44

Vanture A.D., 1992, AJ, 104, 1977

Venn K. A., Irwin M., Shetrone M. D. et al., 2004, AJ, 128, 1177

Wanajo S., Nomoto K., Iwamoto N., Ishimaru Y. \& Beers T. C., 2006, ApJ, 636, 842

Wanajo S., Itoh N., Goriely S., Samyn M., Ishimaru Y., 2005, NuphA, 758, 671

Ward L., Vogel O., Arnesen A., Hallin R., Wannstrom A., 1985, Phys. Scripta 31, 162-165

Warner B., 1968, MNRAS 140, 53-59

Woodward P. R., Herwig F. \& Lin P.-H., 2015, ApJ, 798, 49

Worely C.C., Hill V. J., Sobeck J., Carretta E., 2013, A\&A, 553, A 47

Yang G.C. et al., 2016, RAA, 16, 1

Yanny B. et al., AJ, 2009, 137, 4377

Yong D. et al., 2013, ApJ, 762, 27

Yoon J. et al., 2016, ApJ, 833, 20

York D. et al., AJ, 2000, 120, 1579

Zhao G., Zhao Y., Chu Y., Jing Y. \& Deng L., 2012, RAA, 12, 723

Zijlstra A.A., 2004, MNRAS, 348, 23

\section{APPENDIX}


Table A1 : Equivalent widths (in $\mathrm{m} \AA$ ) of Fe lines used for deriving atmospheric parameters

\begin{tabular}{|c|c|c|c|c|c|c|}
\hline Wavelength $(\AA)$ & $\mathrm{El}$ & $E_{\text {low }}(\mathrm{eV})$ & $\log g f$ & LAMOSTJ091608.81+230734.6 & LAMOSTJ151003.74+305407.3 & Ref \\
\hline 4445.471 & Fe I & 0.087 & -5.380 & $77.50(6.44)$ & - & 1 \\
\hline 4484.220 & & 3.603 & -0.720 & $91.70(6.40)$ & $88.1(5.72)$ & 1 \\
\hline 4489.739 & & 0.121 & -3.966 & $133.5(6.39$ & - & 1 \\
\hline 4619.288 & & 3.603 & -1.120 & $84.2(6.60)$ & $76.7(5.95)$ & 1 \\
\hline 4635.846 & & 2.845 & -2.420 & $56.2(6.45)$ & - & 1 \\
\hline 4637.503 & & 3.283 & -1.390 & $86.3(6.53)$ & - & 1 \\
\hline 4643.464 & & 3.654 & -1.290 & $70.9(6.54)$ & - & 1 \\
\hline 4690.138 & & 3.686 & -1.640 & $53.7(6.60)$ & - & 1 \\
\hline 4882.143 & & 3.417 & -1.640 & $82.1(6.80)$ & $55.7(5.94)$ & 1 \\
\hline 4907.732 & & 3.430 & -1.840 & $61.7(6.62)$ & - & 1 \\
\hline 4908.031 & & 4.217 & -1.396 & $39.8(6.58)$ & - & 2 \\
\hline 4917.229 & & 4.191 & -1.180 & $35.7(6.38)$ & - & 1 \\
\hline 4924.770 & & 2.278 & -2.220 & $111(6.64)$ & $112(5.85)$ & 1 \\
\hline 4939.687 & & 0.859 & -3.340 & $142(6.64)$ & $173(6.02)$ & 1 \\
\hline 4967.890 & & 4.191 & -0.622 & $82.8(6.7)$ & - & 2 \\
\hline 4969.917 & & 4.216 & -0.710 & $56.7(6.31)$ & - & 1 \\
\hline 4985.253 & & 3.930 & -0.560 & $84.8(6.36)$ & - & 2 \\
\hline 5022.236 & & 3.984 & -0.530 & $80.8(6.31)$ & $96(6.05)$ & 1 \\
\hline 5028.126 & & 3.573 & -1.474 & $71.9(6.59)$ & $42.6(5.78)$ & 2 \\
\hline 5049.819 & & 2.278 & -1.420 & $160.4(6.77)$ & - & 1 \\
\hline 5109.652 & & 4.302 & -0.980 & $62.3(6.77)$ & - & 1 \\
\hline 5127.359 & & 0.915 & -3.307 & $133.2(6.37)$ & - & 1 \\
\hline 5159.058 & & 4.283 & -0.820 & $55.3(6.46)$ & - & 1 \\
\hline 5187.915 & & 4.143 & -1.260 & $54.2(6.71)$ & - & 1 \\
\hline 5215.179 & & 3.266 & -0.933 & $121(6.67)$ & - & 1 \\
\hline 5242.491 & & 3.634 & -0.840 & $95.7(6.58)$ & $84.2(5.87)$ & 1 \\
\hline 5247.050 & & 0.087 & -4.946 & - & $136.1(5.89)$ & 1 \\
\hline 5250.209 & & 0.121 & -4.938 & $120.4(6.63)$ & $131.1(5.82)$ & 1 \\
\hline 5253.462 & & 3.283 & -1.670 & $92.1(6.8)$ & $78.6(6.06)$ & 1 \\
\hline 5281.790 & & 3.038 & -1.020 & - & $150.6(5.94)$ & 1 \\
\hline 5322.040 & & 2.280 & -2.840 & $81.2(6.48)$ & $76.4(5.88)$ & 3 \\
\hline 5339.930 & & 3.270 & -0.680 & $132.7(6.63)$ & - & 3 \\
\hline 5364.858 & & 4.445 & 0.2200 & $110.5(6.68)$ & - & 3 \\
\hline 5365.399 & & 3.573 & -1.440 & $86.7(6.8)$ & $55.7(5.9)$ & 2 \\
\hline 5367.479 & & 4.415 & 0.3500 & $113.5(6.57)$ & - & 1 \\
\hline 5369.961 & & 4.370 & 0.3500 & $114.3(6.54)$ & - & 1 \\
\hline 5383.369 & & 4.312 & 0.5000 & $130(6.65)$ & - & 1 \\
\hline 5543.936 & & 4.217 & -1.140 & $58.8(6.74)$ & - & 1 \\
\hline 5569.620 & & 3.420 & -0.490 & $125.7(6.45)$ & - & 3 \\
\hline 5576.090 & & 3.430 & -0.851 & $113.5(6.69)$ & - & 1 \\
\hline 5586.756 & & 3.368 & -0.210 & $157.8(6.71)$ & - & 1 \\
\hline 5618.631 & & 4.209 & -1.380 & $51.4(6.72)$ & - & 1 \\
\hline 5701.544 & & 2.559 & -2.216 & $108.5(6.69)$ & $90.7(5.79)$ & 1 \\
\hline 5741.848 & & 4.256 & -1.730 & $20.8(6.63)$ & - & 1 \\
\hline 5753.120 & & 4.260 & -0.760 & $77.6(6.72)$ & $58.6(6.08)$ & 1 \\
\hline 5856.088 & & 4.294 & -1.640 & $34(6.79)$ & - & 1 \\
\hline 5859.586 & & 4.549 & -0.386 & $83.1(6.79)$ & - & 2 \\
\hline 5862.357 & & 4.549 & -0.051 & $81.7(6.31)$ & - & 2 \\
\hline 5956.692 & & 0.859 & -4.605 & $95.9(6.62)$ & $81.6(5.81)$ & 1 \\
\hline 6003.010 & & 3.881 & -1.120 & - & $47.7(5.82)$ & 1 \\
\hline 6082.710 & & 2.222 & -3.573 & $55.2(6.66)$ & $28.3(5.86)$ & 1 \\
\hline 6136.994 & & 2.198 & -2.950 & $84.5(6.47)$ & $78.3(5.88)$ & 1 \\
\hline 6137.694 & & 2.588 & -1.403 & $157.5(6.76)$ & - & 1 \\
\hline 6151.620 & & 2.180 & -3.290 & $79.8(6.71)$ & $62.4(6.01)$ & 3 \\
\hline 6173.340 & & 2.220 & -2.880 & $101.7(6.72)$ & $108.2(6.17)$ & 3 \\
\hline 6180.204 & & 2.727 & -2.780 & $64.8(6.64)$ & $43.2(5.96)$ & 1 \\
\hline 6200.314 & & 2.608 & -2.437 & - & $85(5.96)$ & 1 \\
\hline 6213.429 & & 2.222 & -2.660 & $113(6.68)$ & $102.7(5.88)$ & 1 \\
\hline 6219.279 & & 2.198 & -2.433 & $120.5(6.56)$ & $140.4(6.08)$ & 1 \\
\hline 6240.646 & & 2.222 & -3.380 & $70.8(6.49)$ & $54.1(5.84)$ & 1 \\
\hline 6252.554 & & 2.404 & -1.687 & $155.1(6.73)$ & $152.2(5.76)$ & 1 \\
\hline 6254.258 & & 2.279 & -2.480 & - & $117.3(5.86)$ & 1 \\
\hline
\end{tabular}


Table A1 continues...

\begin{tabular}{|c|c|c|c|c|c|c|}
\hline Wavelength $(\AA)$ & $\mathrm{El}$ & $E_{\text {low }}(\mathrm{eV})$ & $\log \mathrm{gf}$ & LAMOSTJ091608.81+230734.6 & LAMOSTJ151003.74+305407.3 & Ref \\
\hline 6280.617 & & 0.859 & -4.390 & $120.5(6.75)$ & - & 1 \\
\hline 6297.800 & & 2.222 & -2.740 & $90.3(6.36)$ & - & 1 \\
\hline 6301.500 & & 3.654 & -0.672 & $107.8(6.45)$ & - & 2 \\
\hline 6322.690 & & 2.588 & -2.426 & - & $99.7(6.08)$ & 2 \\
\hline 6335.328 & & 2.198 & -2.230 & $128(6.46)$ & - & 1 \\
\hline 6393.602 & & 2.432 & -1.620 & $158.3(6.71)$ & - & 1 \\
\hline 6408.016 & & 3.686 & -1.048 & $93.5(6.52)$ & - & 2 \\
\hline 6419.950 & & 4.730 & -0.090 & $81.3(6.77)$ & $53.5(6.05)$ & 3 \\
\hline 6421.349 & & 2.278 & -2.027 & - & $153.1(5.91)$ & 1 \\
\hline 6430.850 & & 2.180 & -2.010 & $151.5(6.63)$ & $160.5(5.84)$ & 3 \\
\hline 6481.870 & & 2.278 & -2.984 & $99.5(6.8)$ & - & 1 \\
\hline 6574.227 & & 0.990 & -5.040 & $73.2(6.81)$ & $69.5(6.23)$ & 1 \\
\hline 6575.019 & & 2.588 & -2.820 & $79.4(6.69)$ & - & 1 \\
\hline 6592.910 & & 2.720 & -1.470 & $131.8(6.53)$ & - & 3 \\
\hline 6593.871 & & 2.432 & -2.422 & $107.1(6.55)$ & - & 1 \\
\hline 6677.989 & & 2.692 & -1.470 & $148.4(6.64)$ & $169(6.07)$ & 1 \\
\hline 6739.521 & & 1.557 & -4.950 & $28.6(6.59)$ & - & 1 \\
\hline 6750.150 & & 2.424 & -2.621 & $113.7(6.82)$ & - & 1 \\
\hline 4416.830 & $\mathrm{Fe}$ II & 2.778 & -2.600 & - & $71.3(5.95)$ & 1 \\
\hline 4629.339 & & 2.807 & -2.280 & $108.3(6.55)$ & - & 1 \\
\hline 4923.927 & & 2.891 & -1.320 & $159.8(6.68)$ & - & 1 \\
\hline 5234.620 & & 3.220 & -2.240 & $99.2(6.48)$ & $60.9(5.76)$ & 3 \\
\hline 6247.550 & & 3.890 & -2.340 & $57.1(6.6)$ & $20.3(5.98)$ & 3 \\
\hline 6369.462 & & 2.891 & -4.253 & $29.7(6.81)$ & - & 2 \\
\hline 6456.383 & & 3.903 & -2.075 & $67.3(6.56)$ & $30.8(6.03)$ & 1 \\
\hline
\end{tabular}

The numbers within the parenthesis in columns 5-6 give the derived abundances from the respective line. References: 1. Führ et al. (1988), 2. Kurucz (1988), 3. Lambert et al. (1996)

Table A2 : Equivalent widths (in $\mathrm{mÅ}$ ) of lines used for deriving elemental abundances

\begin{tabular}{|c|c|c|c|c|c|c|}
\hline Wavelength $(\AA)$ & $\mathrm{El}$ & $E_{\text {low }}(\mathrm{eV})$ & $\log g f$ & LAMOSTJ091608.81+230734.6 & LAMOSTJ151003.74+305407.3 & Ref \\
\hline 5682.633 & $\mathrm{Na} \mathrm{I}$ & 2.102 & -0.700 & $109.4(6.15)$ & - & 1 \\
\hline 5688.205 & & 2.100 & -0.450 & $106.8(5.86)$ & - & 1 \\
\hline 6154.226 & & 2.102 & -1.560 & $59.90(6.16)$ & $23.10(5.27)$ & 1 \\
\hline 6160.747 & & 2.104 & -1.260 & $66.40(5.97)$ & $35.20(5.20)$ & 1 \\
\hline 4702.991 & $\mathrm{Mg} \mathrm{I}$ & 4.346 & -0.666 & - & $116.9(5.85)$ & 2 \\
\hline 5528.405 & & 4.346 & -0.620 & $175.6(7.23)$ & $138.9(6.00)$ & 2 \\
\hline 5711.088 & & 4.346 & -1.833 & $92.80(7.01)$ & $64.30(6.20)$ & 2 \\
\hline 5690.425 & Si I & 4.929 & -1.870 & $31.90(6.67)$ & - & 3 \\
\hline 5948.541 & & 5.083 & -1.230 & $61.40(6.72)$ & - & 3 \\
\hline 6145.016 & & 5.616 & -0.820 & $31.60(6.38)$ & - & 4 \\
\hline 4435.679 & $\mathrm{Ca} \mathrm{I}$ & 1.890 & -0.520 & - & $119.2(4.77)$ & 5 \\
\hline 5349.465 & & 2.709 & -1.178 & $38.20(5.42)$ & - & 5 \\
\hline 5512.980 & & 2.932 & -0.290 & $69.90(5.36)$ & - & 5 \\
\hline 5581.965 & & 2.523 & -1.833 & - & $39.60(4.41)$ & 5 \\
\hline 5590.114 & & 2.521 & -0.710 & $89.50(5.67)$ & $35.30(4.33)$ & 5 \\
\hline 5857.451 & & 2.932 & 0.23 & $93.60(5.27)$ & $76.20(4.47)$ & 5 \\
\hline 6102.723 & & 1.879 & -0.890 & $102.0(5.26)$ & $103.5(4.59)$ & 5 \\
\hline 6166.439 & & 2.521 & -0.900 & $64.10(5.33)$ & - & 5 \\
\hline 6169.042 & & 2.523 & -0.550 & $79.50(5.26)$ & - & 5 \\
\hline 6169.563 & & 2.523 & -0.270 & - & $83.30(4.53)$ & 5 \\
\hline 6439.075 & & 2.525 & 0.47 & $157.5(5.64)$ & - & 5 \\
\hline 6449.808 & & 2.523 & -0.550 & $86.90(5.37)$ & $70.30(4.61)$ & 5 \\
\hline 6471.662 & & 2.525 & -0.590 & - & $56.80(4.48)$ & 5 \\
\hline 6493.781 & & 2.521 & 0.14 & $117.9(5.27)$ & - & 5 \\
\hline
\end{tabular}

The numbers within the parenthesis in columns 5-6 give the derived abundances from the respective line. 
Table A2 continues...

\begin{tabular}{|c|c|c|c|c|c|c|}
\hline Wavelength $(\AA)$ & $\mathrm{El}$ & $E_{l o w}(\mathrm{eV})$ & $\log g f$ & LAMOSTJ091608.81+230734.6 & LAMOSTJ151003.74+305407.3 & Ref \\
\hline 4512.734 & $\mathrm{Ti} \mathrm{I}$ & 0.836 & -0.480 & $84.90(4.04)$ & - & 6 \\
\hline 4617.269 & & 1.749 & 0.389 & $87.00(4.29)$ & - & 6 \\
\hline 4759.272 & & 2.255 & 0.514 & $40.30(3.82)$ & - & 6 \\
\hline 4840.874 & & 0.899 & -0.509 & $81.20(3.98)$ & $78.00(3.19)$ & 6 \\
\hline 5007.210 & & 0.820 & 0.1700 & $131.4(4.25)$ & - & 6 \\
\hline 5024.842 & & 0.818 & -0.602 & $82.10(3.95)$ & $90.20(3.30)$ & 6 \\
\hline 5210.386 & & 0.047 & -0.884 & $110.0(3.79)$ & $143.9(3.23)$ & 6 \\
\hline 5460.499 & & 0.050 & -2.880 & $27.30(4.26)$ & - & 7 \\
\hline 5918.535 & & 1.070 & -1.460 & $33.30(4.16)$ & $25.70(3.50)$ & 6 \\
\hline 5922.110 & & 1.046 & -1.466 & $35.10(4.17)$ & - & 6 \\
\hline 5941.751 & & 1.050 & -1.510 & $30.70(4.14)$ & - & 6 \\
\hline 6303.757 & & 1.443 & -1.566 & $25.00(4.03)$ & - & 6 \\
\hline 4568.314 & Ti II & 1.220 & -2.650 & $69.10(3.98)$ & $30.20(3.02)$ & 6 \\
\hline 4571.960 & & 1.571 & -0.530 & - & $140.4(3.16)$ & 6 \\
\hline 4764.526 & & 1.236 & -2.770 & - & $49.90(3.47)$ & 6 \\
\hline 4798.521 & & 1.080 & -2.430 & $72.10(3.62)$ & - & 6 \\
\hline 4865.612 & & 1.116 & -2.610 & $71.30(3.82)$ & - & 6 \\
\hline 5185.900 & & 1.890 & -1.350 & $82.10(3.66)$ & $86.30(3.34)$ & 6 \\
\hline 5381.015 & & 1.566 & -2.080 & - & $46.00(3.07)$ & 6 \\
\hline 5247.565 & Cr I & 0.961 & -1.640 & - & $81.80(3.60)$ & 6 \\
\hline 5296.691 & & 0.982 & -1.400 & $131.8(5.17)$ & - & 6 \\
\hline 5300.744 & & 0.982 & -2.120 & - & $49.00(3.68)$ & 6 \\
\hline 5345.801 & & 1.003 & -0.980 & $150.1(5.12)$ & $128.7(3.64)$ & 6 \\
\hline 5348.312 & & 1.003 & -1.290 & $121.6(4.86)$ & $106.5(3.62)$ & 6 \\
\hline 5409.772 & & 1.030 & -0.720 & $156.1(4.98)$ & $130.0(3.41)$ & 6 \\
\hline 5787.965 & & 3.323 & -0.083 & $67.70(5.30)$ & - & 6 \\
\hline 6362.862 & & 0.941 & -3.623 & $33.00(5.33)$ & - & 5 \\
\hline 4686.207 & $\mathrm{Ni} \mathrm{I}$ & 3.597 & -0.640 & $64.70(5.44)$ & - & 5 \\
\hline 4752.415 & & 3.658 & -0.700 & - & $40.90(4.76)$ & 8 \\
\hline 4821.130 & & 4.153 & -0.850 & $37.40(5.73)$ & - & 8 \\
\hline 4953.200 & & 3.74 & -0.670 & $59.80(5.51)$ & $22.00(4.45)$ & 5 \\
\hline 4980.166 & & 3.606 & -0.110 & - & $72.90(4.55)$ & 8 \\
\hline 5082.350 & & 3.657 & -0.540 & $77.40(5.63)$ & $36.50(4.49)$ & 8 \\
\hline 5102.960 & & 1.676 & -2.620 & - & $93.90(4.87)$ & 8 \\
\hline 6086.280 & & 4.266 & -0.530 & $53.50(5.79)$ & - & 8 \\
\hline 6175.360 & & 4.089 & -0.530 & $52.30(5.55)$ & - & 8 \\
\hline 6177.236 & & 1.826 & -3.500 & $38.40(5.56)$ & - & 8 \\
\hline 6186.710 & & 4.106 & -0.777 & $41.80(5.62)$ & - & 8 \\
\hline 6204.600 & & 4.088 & -1.130 & $32.40(5.76)$ & - & 8 \\
\hline 6327.593 & & 1.676 & -3.150 & $79.10(5.72)$ & - & 8 \\
\hline 6378.247 & & 4.154 & -0.890 & $31.40(5.57)$ & - & 5 \\
\hline 6643.629 & & 1.676 & -2.300 & - & $106.6(4.53)$ & 1 \\
\hline 4722.150 & Zn I & 4.029 & -0.370 & - & $34.80(2.66)$ & 9 \\
\hline 4810.530 & & 4.080 & -0.170 & - & $33.80(2.46)$ & 9 \\
\hline 6362.338 & & 0.150 & 5.796 & $29.60(4.11)$ & - & 10 \\
\hline 4607.327 & Sr I & 0.000 & -0.570 & $84.20(3.21)$ & - & 11 \\
\hline 6435.004 & Y I & 0.066 & -0.820 & $47.70(2.73)$ & $55.20(2.19)$ & 12 \\
\hline 4883.684 & Y II & 1.084 & 0.07 & $145.4(2.37)$ & - & 12 \\
\hline 5119.112 & & 0.992 & -1.360 & - & $172.7(3.13)$ & 12 \\
\hline 5289.815 & & 1.033 & -1.850 & $63.50(2.27)$ & - & 12 \\
\hline 5402.774 & & 1.839 & -0.510 & $77.50(2.20)$ & - & 13 \\
\hline 5544.611 & & 1.738 & -1.090 & $64.50(2.36)$ & - & 12 \\
\hline 5546.009 & & 1.748 & -1.110 & - & $124.9(2.97)$ & 12 \\
\hline 5662.925 & & 1.944 & 0.16 & $105.3(2.26)$ & - & 13 \\
\hline 6613.733 & & 1.748 & -1.100 & $74.20(2.49)$ & - & 12 \\
\hline
\end{tabular}

The numbers within the parenthesis in columns 5-6 give the derived abundances from the respective line. 
Table A2 continues...

\begin{tabular}{|c|c|c|c|c|c|c|}
\hline Wavelength $(\AA)$ & $\mathrm{El}$ & $E_{\text {low }}(\mathrm{eV})$ & $\log g f$ & LAMOSTJ091608.81+230734.6 & LAMOSTJ151003.74+305407.3 & Ref \\
\hline 4739.480 & Zr I & 0.651 & 0.230 & $84.30(3.30)$ & $47.30(1.89)$ & 14 \\
\hline 4772.323 & & 0.623 & 0.044 & $60.90(2.92)$ & $63.90(2.27)$ & 14 \\
\hline 4805.889 & & 0.687 & -0.420 & $40.40(3.06)$ & - & 14 \\
\hline 6134.585 & & 0.000 & -1.280 & $54.10(3.18)$ & $32.50(2.15)$ & 14 \\
\hline 4257.120 & Ce II & 0.460 & -1.116 & - & $36.80(1.15)$ & 15 \\
\hline 4336.244 & & 0.704 & -0.564 & $82.70(2.32)$ & - & 15 \\
\hline 4349.789 & & 0.701 & -0.107 & - & $65.90(1.34)$ & 13 \\
\hline 4407.273 & & 0.701 & -0.741 & - & $53.70(1.32)$ & 15 \\
\hline 4497.846 & & 0.958 & -0.349 & - & $67.00(1.44)$ & 15 \\
\hline 4508.079 & & 0.621 & -1.238 & $60.90(2.28)$ & - & 15 \\
\hline 4628.161 & & 0.516 & 0.008 & $120.8(2.43)$ & - & 15 \\
\hline 4747.167 & & 0.320 & -1.246 & - & $50.80(1.25)$ & 15 \\
\hline 4873.999 & & 1.107 & -0.892 & $49.60(2.22)$ & - & 15 \\
\hline 5187.458 & & 1.211 & -0.104 & $86.20(2.35)$ & - & 15 \\
\hline 5274.229 & & 1.044 & -0.323 & - & $96.10(1.21)$ & 6 \\
\hline 5330.556 & & 0.869 & -0.760 & $72.30(2.23)$ & $59.40(1.53)$ & 15 \\
\hline 6034.205 & & 1.458 & -1.019 & $42.10(2.52)$ & - & 15 \\
\hline 5188.217 & Pr II & 0.922 & -1.145 & $26.00(1.59)$ & $20.40(1.18)$ & 15 \\
\hline 5219.045 & & 0.795 & -0.240 & - & $77.20(1.02)$ & 16 \\
\hline 5259.728 & & 0.633 & -0.682 & $65.90(1.60)$ & $61.90(1.04)$ & 16 \\
\hline 5292.619 & & 0.648 & -0.300 & $83.40(1.62)$ & - & 16 \\
\hline 5322.772 & & 0.482 & -0.315 & $90.30(1.58)$ & $98.00(0.98)$ & 15 \\
\hline 6165.891 & & 0.923 & -0.205 & $59.20(1.24)$ & $63.00(0.87)$ & 15 \\
\hline 6278.676 & & 1.196 & -0.630 & $22.20(1.23)$ & - & 16 \\
\hline 4446.384 & Nd II & 0.204 & 0.590 & - & $110.9(1.27)$ & 17 \\
\hline 4451.563 & & 0.380 & -0.040 & $115.1(2.04)$ & - & 17 \\
\hline 4556.133 & & 0.064 & -1.610 & $76.60(2.13)$ & - & 15 \\
\hline 4811.342 & & 0.064 & -1.140 & $92.80(2.00)$ & - & 15 \\
\hline 4825.478 & & 0.182 & -0.860 & $112.4(2.37)$ & - & 15 \\
\hline 4947.020 & & 0.559 & -1.250 & - & $58.00(1.42)$ & 15 \\
\hline 4961.387 & & 0.631 & -0.710 & $96.00(2.32)$ & $98.20(1.56)$ & 15 \\
\hline 5212.361 & & 0.204 & -0.870 & - & $104.7(1.21)$ & 15 \\
\hline 5276.869 & & 0.859 & -0.440 & - & $71.80(1.15)$ & 17 \\
\hline 5287.133 & & 0.744 & -1.300 & - & $39.30(1.39)$ & 15 \\
\hline 5356.967 & & 1.264 & -0.250 & - & $68.20(1.42)$ & 17 \\
\hline 5361.510 & & 0.680 & -0.400 & - & $92.40(1.16)$ & 17 \\
\hline 5442.264 & & 0.680 & -0.910 & $84.40(2.19)$ & $86.00(1.56)$ & 17 \\
\hline 5485.696 & & 1.264 & -0.120 & $77.70(1.96)$ & $68.70(1.28)$ & 17 \\
\hline 5603.648 & & 0.380 & -1.830 & $71.70(2.43)$ & - & 15 \\
\hline 5718.118 & & 1.410 & -0.340 & - & $48.90(1.39)$ & 17 \\
\hline 5825.857 & & 1.080 & -0.760 & $65.90(2.08)$ & $50.00(1.40)$ & 15 \\
\hline 4458.509 & Sm II & 0.104 & -1.110 & $76.30(1.45)$ & - & 15 \\
\hline 4499.475 & & 0.248 & -1.413 & $52.10(1.34)$ & $61.20(1.04)$ & 15 \\
\hline 4519.630 & & 0.543 & -0.751 & - & $81.70(1.07)$ & 15 \\
\hline 4566.210 & & 0.330 & -1.245 & $57.80(1.38)$ & $63.70(1.01)$ & 15 \\
\hline 4615.444 & & 0.544 & -1.262 & $50.60(1.49)$ & - & 15 \\
\hline 4642.228 & & 0.379 & -0.951 & $71.20(1.45)$ & $88.90(1.15)$ & 15 \\
\hline 4674.593 & & 0.184 & -1.055 & $68.10(1.23)$ & - & 15 \\
\hline 4676.902 & & 0.040 & -1.407 & $62.30(1.28)$ & - & 15 \\
\hline 4704.400 & & 0.000 & -1.562 & $73.10(1.62)$ & $65.00(0.89)$ & 15 \\
\hline 4726.026 & & 0.333 & -1.849 & $31.80(1.42)$ & $27.70(1.02)$ & 15 \\
\hline 4854.368 & & 0.379 & -1.873 & $32.10(1.49)$ & - & 15 \\
\hline
\end{tabular}

The numbers within the parenthesis in columns 5-6 give the derived abundances from the respective line.

References: 1. Kurucz et al. (1975), 2. Lincke et al. (1971), 3. Garz (1973), 4. Schulz-Gulde (1969), 5. Kurucz (1988), 6. Martin et al. (1988), 7. Smith et al. (1978), 8. Führ et al. (1988), 9. Warner (1968), 10. Lambert et al. (1969), 11. Corliss et al. (1962), 12. Hannaford et al. (1982), 13. Cowley et al. (1983), 14. Biemont et al. (1981), 15. Meggers et al. (1975), 16. Lagehaling et al. (1976), 17. Ward et al. 